\title{
Triebel-Lizorkin space boundedness of rough singular integrals associated to surfaces
}

Kôzô Yabuta*

\section{"Correspondence:}

kyabuta3@kwansei.ac.jp

Research Center for Mathematical

Sciences, Kwansei Gakuin University,

Gakuen 2-1, Sanda, 669-1337, Japan

\begin{abstract}
In the present paper, we consider the boundedness of the rough singular integral operator $T_{\Omega, h, \phi}$ along a surface $\Gamma=\{x=\phi(|y|) y /|y|\}$ on the Triebel-Lizorkin space $\dot{F}_{p, q}^{\alpha}\left(\mathbb{R}^{n}\right)$ for $\Omega \in H^{1}\left(S^{n-1}\right)$ and $\Omega$ belonging to some class $W \mathcal{F}_{\alpha}\left(S^{n-1}\right)$, which relates to the Grafakos-Stefanov class.
\end{abstract}

MSC: Primary 42B20; secondary 42B25; 47G10

Keywords: singular integrals; Triebel-Lizorkin spaces; rough kernel

\section{Introduction}

Let $\mathbb{R}^{n}(n \geq 2)$ be the $n$-dimensional Euclidean space and $S^{n-1}$ be the unit sphere in $\mathbb{R}^{n}$ equipped with the induced Lebesgue measure $d \sigma=d \sigma(\cdot)$. Suppose that $\Omega \in L^{1}\left(S^{n-1}\right)$ satisfies the cancelation condition

$$
\int_{S^{n-1}} \Omega\left(y^{\prime}\right) d \sigma\left(y^{\prime}\right)=0 .
$$

For a suitable function $\phi$ and a measurable function $h$ on $[0, \infty)$, we denote by $T_{\Omega, \phi, h}$ the singular integral operator along the surface

$$
\Gamma=\left\{x=\phi(|y|) y^{\prime}: y \in \mathbb{R}^{n}\right\}
$$

defined as follows:

$$
T_{\Omega, h, \phi} f(x)=\text { p.v. } \int_{\mathbb{R}^{n}} \frac{h(|y|) \Omega\left(y^{\prime}\right)}{|y|^{n}} f\left(x-\phi(|y|) y^{\prime}\right) d y
$$

for $f$ in the Schwartz class $\mathcal{S}\left(\mathbb{R}^{n}\right)$. If $\phi=1$, then $T_{\Omega, h, \phi}$ is the classical singular integral operator $T_{\Omega, h}$, which is defined by

$$
T_{\Omega, h} f(x)=\text { p.v. } \int_{\mathbb{R}^{n}} \frac{h(|y|) \Omega\left(y^{\prime}\right)}{|y|^{n}} f(x-y) d y .
$$

When $h \equiv 1$, we denote simply $T_{\Omega, h, \phi}$ and $T_{\Omega, h}$ by $T_{\Omega, \phi}$ and $T_{\Omega}$, respectively.

The $L^{p}$ boundedness of singular integrals along the surface has attracted the attention of many authors [1-3], etc. There are several papers concerning rough kernels associated to surfaces as above [4-6]. As one of them, we count the following one.

C) 2015 Yabuta; licensee Springer. This is an Open Access article distributed under the terms of the Creative Commons Attribution License (http://creativecommons.org/licenses/by/4.0), which permits unrestricted use, distribution, and reproduction in any medium, provided the original work is properly credited. 
Theorem A ([5]) Let $h \in \Delta_{\gamma}$ for some $\gamma \geq 2,1<p<\infty, \Omega \in H^{1}\left(S^{n-1}\right)$. Let $\phi$ be a nonnegative $C^{1}$ function on $(0, \infty)$ satisfying

(i) $\phi(t)$ is strictly increasing and $\phi(2 t) \geq \lambda \phi(t)$ for all $t>0$ and some $\lambda>1$,

(ii) $\phi(t)$ satisfies a doubling condition $\phi(2 t) \leq c \phi(t)$ for all $t>0$ and some $c>1$,

(iii) $\phi^{\prime}(t) \geq C_{1} \phi(t) / t$ for all $t>0$ and some $C_{1}$.

Then $T_{\Omega, h, \phi}$ is bounded on $L^{p}\left(\mathbb{R}^{n}\right)$.

This is, in fact, stated in the more general setting, i.e., for a weighted case (Theorem 1 and Corollary 1 in [5]), but we state this as above for our purpose and for the sake of simplicity. We note here that condition (i) follows from (iii).

On the other hand, Triebel-Lizorkin space boundedness of rough singular integrals was also investigated by many authors, see [7, 8] and [9].

Before stating the following result, let us recall the definitions of some function spaces. First we give the definition of the Hardy space $H^{1}\left(S^{n-1}\right)$ :

$$
\begin{aligned}
& H^{1}\left(S^{n-1}\right) \\
& \quad=\left\{\omega \in L^{1}\left(S^{n-1}\right)\left|\|f\|_{H^{1}\left(S^{n-1}\right)}=\left\|\sup _{0 \leq r<1}\left|\int_{S^{n-1}} \omega\left(y^{\prime}\right) P_{r(\cdot)}\left(y^{\prime}\right) d \sigma\left(y^{\prime}\right)\right|\right\|_{L^{1}\left(S^{n-1}\right)}<\infty\right\},\right.
\end{aligned}
$$

where $P_{r y^{\prime}}\left(x^{\prime}\right)$ denotes the Poisson kernel on $S^{n-1}$ defined by

$$
P_{r y^{\prime}}\left(x^{\prime}\right)=\frac{1-r^{2}}{\left|r y^{\prime}-x^{\prime}\right|^{n}}, \quad 0 \leq r<1 \text { and } x^{\prime}, y^{\prime} \in S^{n-1} .
$$

For $1 \leq \gamma \leq \infty, \Delta_{\gamma}\left(\mathbb{R}_{+}\right)$is the collection of all measurable functions $h:[0, \infty) \rightarrow \mathbb{C}$ satisfying

$$
\|h\|_{\Delta_{\gamma}}=\sup _{R>0}\left(\frac{1}{R} \int_{0}^{R}|h(t)|^{\gamma} d t\right)^{1 / \gamma}<\infty .
$$

Note that

$$
L^{\infty}\left(\mathbb{R}_{+}\right)=\Delta_{\infty}\left(\mathbb{R}_{+}\right) \subset \Delta_{\beta}\left(\mathbb{R}_{+}\right) \subset \Delta_{\alpha}\left(\mathbb{R}_{+}\right) \quad \text { for } \alpha<\beta,
$$

and all these inclusions are proper.

As a result of boundedness on Triebel-Lizorkin spaces, we cite the following one, which is somewhat different from our setting, but closely related.

Theorem B ([9]) Let $\Omega \in H^{1}\left(S^{n-1}\right)$ satisfy the cancelation condition (1.1) and $h \in \Delta_{\gamma}$ for some $1<\gamma \leq \infty$. Let $P=\left(P_{1}, P_{2}, \ldots, P_{d}\right)$ be real polynomials in $y$. Then, for the singular integral

$$
T_{\Omega, P, h} f(x)=\text { p.v. } \int_{\mathbb{R}^{n}} \frac{h(|y|) \Omega\left(y^{\prime}\right)}{|y|^{n}} f(x-P(y)) d y
$$

(i) for $\alpha \in \mathbb{R}$ and $\left|\frac{1}{p}-\frac{1}{2}\right|<\min \left(\frac{1}{2}, \frac{1}{\gamma^{\prime}}\right)$ and $\left|\frac{1}{q}-\frac{1}{2}\right|<\min \left(\frac{1}{2}, \frac{1}{\gamma^{\prime}}\right)$, there exists a constant $C>0$ such that $\left\|T_{\Omega, P, h} f\right\|_{\dot{F}_{p, q}^{\alpha}\left(\mathbb{R}^{d}\right)} \leq C\|f\|_{\dot{F}_{p, q}^{\alpha}\left(\mathbb{R}^{d}\right)} ;$ 
(ii) for $\alpha \in \mathbb{R}$ and $\left|\frac{1}{p}-\frac{1}{2}\right|<\min \left(\frac{1}{2}, \frac{1}{\gamma^{\prime}}\right)$ and $1<q<\infty$, there exists a constant $C>0$ such that $\left\|T_{\Omega, P, h} f\right\|_{\dot{B}_{p, q}^{\alpha}\left(\mathbb{R}^{d}\right)} \leq C\|f\|_{\dot{B}_{p, q}^{\alpha}\left(\mathbb{R}^{d}\right)}$.

Remark 1 We think that there is a gap in the proof of part (i) in the above theorem. Their proof works in the same region as in our Theorem 1.1 below.

Besides $H^{1}\left(S^{n-1}\right)$, there is another class of kernels which leads to $L^{p}$ and Triebel-Lizorkin space boundedness of singular integral operators $T_{\Omega, h}$. It is closely related to the class $\mathcal{F}_{\alpha}$ introduced by Grafakos and Stefanov [10]. We say $\Omega \in W \mathcal{F}_{\beta}=W \mathcal{F}_{\beta}\left(S^{n-1}\right)$ if

$$
\begin{aligned}
\|\Omega\|_{W \mathcal{F}_{\beta}} & :=\sup _{\xi^{\prime} \in S^{n-1}}\left(\int_{S^{n-1}} \int_{S^{n-1}}\left|\Omega\left(y^{\prime}\right) \Omega\left(z^{\prime}\right)\right| \log ^{\beta} \frac{2 e}{\left|\left(y^{\prime}-z^{\prime}\right) \cdot \xi^{\prime}\right|} d \sigma\left(y^{\prime}\right) d \sigma\left(z^{\prime}\right)\right)^{\frac{1}{2}} \\
& <\infty
\end{aligned}
$$

We note that $\bigcup_{r>1} L^{r}\left(S^{n-1}\right) \subset W \mathcal{F}_{\beta_{2}}\left(S^{n-1}\right) \subset W \mathcal{F}_{\beta_{1}}\left(S^{n-1}\right)$ for $0<\beta_{1}<\beta_{2}<\infty$.

About the inclusion relation between $\mathcal{F}_{\beta_{1}}\left(S^{n-1}\right)$ and $W \mathcal{F}_{\beta_{2}}\left(S^{n-1}\right)$, the following is known: when $n=2$, Lemma 1 in [11] shows $\mathcal{F}_{\beta}\left(S^{1}\right) \subset W \mathcal{F}_{\beta}\left(S^{1}\right)$. It is also known that $W_{\mathcal{F}_{2 \alpha}}\left(S^{1}\right) \backslash$ $\left(\mathcal{F}_{\alpha}\left(S^{1}\right) \cup H^{1}\left(S^{1}\right)\right) \neq \emptyset, c f .[12]$.

Theorem C ([12]) Let $h \in \Delta_{\gamma}$ for some $1<\gamma \leq \infty$. Suppose that $\Omega \in W \mathcal{F}_{\beta}=W \mathcal{F}_{\beta}\left(S^{n-1}\right)$ for some $\beta>\max \left(\gamma^{\prime}, 2\right)$, and it satisfies the cancelation condition (1.1). Then the singular integral operator $T_{\Omega, h}$ is bounded on $\dot{F}_{p, q}^{\alpha}\left(\mathbb{R}^{n}\right)$ if $\alpha \in \mathbb{R}$, and $(1 / p, 1 / q)$ belongs to the interior of the parallelogram $P_{1} P_{2} P_{3} P_{4}$, where $P_{1}=\left(\frac{\max \left(\gamma^{\prime}, 2\right)}{2 \beta}, \frac{\max \left(\gamma^{\prime}, 2\right)}{2 \beta}\right), P_{2}=\left(\frac{1}{\gamma^{\prime}}+\frac{\max \left(\gamma^{\prime}, 2\right)}{2 \beta}\left(\frac{1}{\gamma}-\right.\right.$ $\left.\left.\frac{1}{\gamma^{\prime}}\right), \frac{\max \left(\gamma^{\prime}, 2\right)}{2 \beta}\right), P_{3}=\left(1-\frac{\max \left(\gamma^{\prime}, 2\right)}{2 \beta}, 1-\frac{\max \left(\gamma^{\prime}, 2\right)}{2 \beta}\right)$, and $P_{4}=\left(\frac{1}{\gamma}-\frac{\max \left(\gamma^{\prime}, 2\right)}{2 \beta}\left(\frac{1}{\gamma}-\frac{1}{\gamma^{\prime}}\right), 1-\frac{\max \left(\gamma^{\prime}, 2\right)}{2 \beta}\right)$.

Let us recall the definitions of the homogeneous Triebel-Lizorkin spaces $\dot{F}_{p, q}^{\alpha}=\dot{F}_{p, q}^{\alpha}\left(\mathbb{R}^{n}\right)$ and the homogeneous Besov spaces $\dot{B}_{p, q}^{\alpha}=\dot{B}_{p, q}^{\alpha}\left(\mathbb{R}^{n}\right)$. For $0<p, q \leq \infty(p \neq \infty)$ and $\alpha \in \mathbb{R}$, $\dot{F}_{p, q}^{\alpha}\left(\mathbb{R}^{n}\right)$ is defined by

$$
\dot{F}_{p, q}^{\alpha}\left(\mathbb{R}^{n}\right)=\left\{f \in \mathcal{S}^{\prime}\left(\mathbb{R}^{n}\right):\|f\|_{\dot{F}_{p, q}^{\alpha}}=\left\|\left(\sum_{k \in \mathbb{Z}} 2^{k \alpha q}\left|\Psi_{k} * f\right|^{q}\right)^{1 / q}\right\|_{L^{p}}<\infty\right\}
$$

and $\dot{B}_{p}^{\alpha, q}\left(\mathbb{R}^{n}\right)$ is defined by

$$
\dot{B}_{p, q}^{\alpha}\left(\mathbb{R}^{n}\right)=\left\{f \in \mathcal{S}^{\prime}\left(\mathbb{R}^{n}\right):\|f\|_{\dot{B}_{p, q}^{\alpha}}=\left(\sum_{k \in \mathbb{Z}} 2^{k \alpha q}\left\|\Psi_{k} * f\right\|_{L^{p}}^{q}\right)^{1 / q}<\infty\right\},
$$

where $\mathcal{S}^{\prime}\left(\mathbb{R}^{n}\right)$ denotes the tempered distribution class on $\mathbb{R}^{n}, \widehat{\Psi}_{k}(\xi)=\Phi\left(2^{-k} \xi\right)$ for $k \in \mathbb{Z}$ and $\Phi \in C_{c}^{\infty}\left(\mathbb{R}^{n}\right)$ is a radial function satisfying the following conditions:

(i) $0 \leq \Phi \leq 1$;

(ii) $\operatorname{supp} \Phi \subset\{\xi: 1 / 2 \leq|\xi| \leq 2\}$;

(iii) $\Phi>c>0 \quad$ if $3 / 5 \leq|\xi| \leq 5 / 3$;

(iv) $\sum_{j \in \mathbb{Z}} \Phi\left(2^{-j} \xi\right)=1 \quad(\xi \neq 0)$. 
The inhomogeneous versions of Triebel-Lizorkin space and Besov space, which are denoted by $F_{p, q}^{\alpha}\left(\mathbb{R}^{n}\right)$ and $B_{p, q}^{\alpha}\left(\mathbb{R}^{n}\right)$ respectively, are obtained by adding the term $\left\|\Phi_{0} * f\right\|_{p}$ to the right-hand side of (1.5) or (1.6) with $\sum_{k \in \mathbb{Z}}$ replaced by $\sum_{k=0}^{\infty}$, where $\Phi_{0} \in \mathcal{S}\left(\mathbb{R}^{n}\right)$, $\operatorname{supp} \widehat{\Phi}_{0} \subset\{\xi:|\xi| \leq 2\}$, and $\widehat{\Phi}_{0}(\xi)>c>0$ if $|\xi| \leq 5 / 3$.

The following properties of the Triebel-Lizorkin space and the Besov space are well known. Let $1<p, q<\infty, \alpha \in \mathbb{R}$, and $1 / p+1 / p^{\prime}=1,1 / q+1 / q^{\prime}=1$ :

(a) $\quad \dot{F}_{2,2}^{0}=\dot{B}_{2,2}^{0}=L^{2}, \quad \dot{F}_{p, 2}^{0}=L^{p} \quad$ and $\dot{F}_{p, p}^{\alpha}=\dot{B}_{p, p}^{\alpha} \quad$ for $1<p<\infty$ and $\dot{F}_{\infty, 2}^{0}=\mathrm{BMO}$;

(b) $F_{p, q}^{\alpha} \sim \dot{F}_{p, q}^{\alpha} \cap L^{p} \quad$ and $\|f\|_{F_{p, q}^{\alpha}} \sim\|f\|_{\dot{F}_{p, q}^{\alpha}}+\|f\|_{L^{p}} \quad(\alpha>0)$;

(c) $B_{p, q}^{\alpha} \sim \dot{B}_{p, q}^{\alpha} \cap L^{p} \quad$ and $\|f\|_{B_{p, q}^{\alpha}} \sim\|f\|_{\dot{B}_{p, q}^{\alpha}}+\|f\|_{L^{p}} \quad(\alpha>0)$;

(d) $\left(\dot{F}_{p, q}^{\alpha}\right)^{*}=\dot{F}_{p^{\prime}, q^{\prime}}^{-\alpha}$ and $\left(F_{p, q}^{\alpha}\right)^{*}=F_{p^{\prime}, q^{\prime}}^{-\alpha}$;

(e) $\left(\dot{B}_{p, q}^{\alpha}\right)^{*}=\dot{B}_{p^{\prime}, q^{\prime}}^{-\alpha}$ and $\left(B_{p, q}^{\alpha}\right)^{*}=B_{p^{\prime}, q^{\prime}}^{-\alpha}$;

(f) $\left(\dot{F}_{p, q_{1}}^{\alpha_{1}}, \dot{F}_{p, q_{2}}^{\alpha_{2}}\right)_{\theta, q}=\dot{B}_{p, q}^{\alpha}$

$$
\left(\alpha_{1} \neq \alpha_{2}, 0<p<\infty, 0<q, q_{1}, q_{2} \leq \infty, \alpha=(1-\theta) \alpha_{1}+\theta \alpha_{2}, 0<\theta<1\right) .
$$

See [13] and [14] for more properties of $\dot{F}_{p, q}^{\alpha}$ and $\dot{B}_{p, q}^{\alpha}$. See Triebel [14], p.64 and p.244, for (f).

Now we can state our first result.

Theorem 1.1 Let $\phi$ be a positive increasing function on $(0, \infty)$ satisfying

$$
\phi(2 t) \leq c_{1} \phi(t) \quad(t>0) \text { for some } c_{1}>1
$$

and

$$
\varphi(t)=\phi(t) /\left(t \phi^{\prime}(t)\right) \in L^{\infty}(0, \infty)
$$

Let $h \in \Delta_{\gamma}$ for some $1<\gamma \leq \infty$. Suppose $\Omega \in H^{1}\left(S^{n-1}\right)$ satisfying the cancelation condition (1.1). Then

(i) $T_{\Omega, h, \phi}$ is bounded on $\dot{F}_{p, q}^{\alpha}\left(\mathbb{R}^{n}\right)$ for $\alpha \in \mathbb{R}$ and $p$, $q$ with $\left(\frac{1}{p}, \frac{1}{q}\right)$ belonging to the interior of the octagon $P_{1} P_{2} R_{2} P_{3} P_{4} P_{5} R_{4} P_{6}$ (hexagon $P_{1} P_{2} P_{3} P_{4} P_{5} P_{6}$ in the case $1<\gamma \leq 2$ ), where $P_{1}=\left(\frac{1}{2}-\frac{1}{\max \left\{2, \gamma^{\prime}\right\}}, \frac{1}{2}-\frac{1}{\max \left\{2, \gamma^{\prime}\right\}}\right), P_{2}=\left(\frac{1}{2}, \frac{1}{2}-\frac{1}{\max \left\{2, \gamma^{\prime}\right\}}\right), P_{3}=\left(\frac{1}{2}+\frac{1}{\max \left\{2, \gamma^{\prime}\right\}}, \frac{1}{2}\right)$, $P_{4}=\left(\frac{1}{2}+\frac{1}{\max \left\{2, \gamma^{\prime}\right\}}, \frac{1}{2}+\frac{1}{\max \left\{2, \gamma^{\prime}\right\}}\right), P_{5}=\left(\frac{1}{2}, \frac{1}{2}+\frac{1}{\max \left\{2, \gamma^{\prime}\right\}}\right), P_{6}=\left(\frac{1}{2}-\frac{1}{\max \left\{2, \gamma^{\prime}\right\}}, \frac{1}{2}\right)$, $R_{2}=\left(1-\frac{1}{2 \gamma}, \frac{1}{2 \gamma}\right)$, and $R_{4}=\left(\frac{1}{2 \gamma}, 1-\frac{1}{2 \gamma}\right)$;

(ii) $T_{\Omega, h, \phi}$ is bounded on $\dot{B}_{p, q}^{\alpha}\left(\mathbb{R}^{n}\right)$ for $\alpha \in \mathbb{R}$ and $p$, q satisfying $\left|\frac{1}{2}-\frac{1}{p}\right|<\min \left\{\frac{1}{2}, \frac{1}{\gamma^{\prime}}\right\}$ and $1<q<\infty$.

See Figures 1 and 2 for the conclusion (i) of Theorem 1.1.

Example 1 As typical examples of $\phi$ satisfying conditions (1.9) and (1.10), we list the following three: $t^{\alpha} \log ^{\beta}(1+t)(\alpha>0, \beta \geq 0),\left(2 t^{2}-2 t+1\right) t^{1+\alpha}(\alpha \geq 0)$, and $\phi(t)=2 t^{2}+t$ $\left(0<t<\frac{\pi}{2}\right), \phi(t)=2 t^{2}+t \sin t\left(t \geq \frac{\pi}{2}\right)$. Note that linear combinations with positive coefficients of functions $\phi$ 's satisfying the above two conditions also satisfy them, $c f$. [15]. 


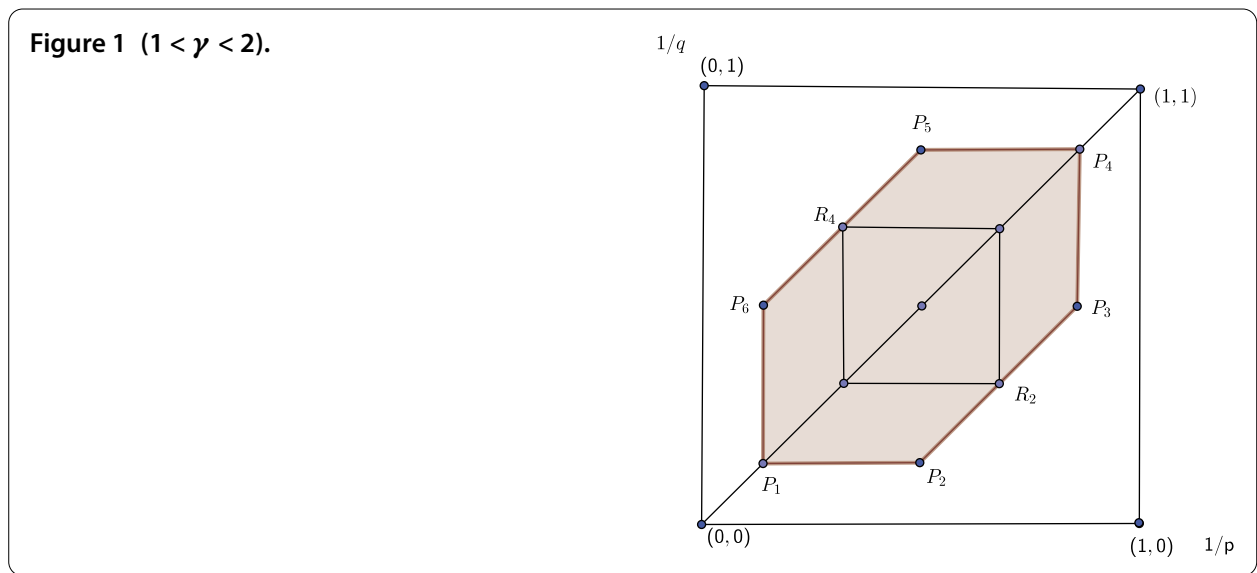

Figure $2(2 \leq \gamma \leq \infty)$.

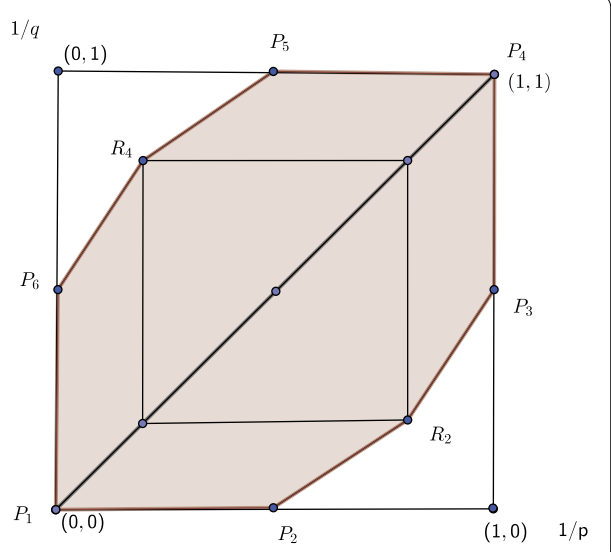

We shall state our following result, which relates to two function spaces $L(\log L)\left(S^{n-1}\right)$ and the block spaces $B_{q}^{(0,0)}\left(S^{n-1}\right)$. Let $L(\log L)^{\alpha}\left(S^{n-1}\right)$ (for $\left.\alpha>0\right)$ denote the class of all measurable functions $\Omega$ on $S^{n-1}$ which satisfy

$$
\|\Omega\|_{L(\log L)^{\alpha}\left(S^{n-1}\right)}=\int_{S^{n-1}}\left|\Omega\left(y^{\prime}\right)\right| \log ^{\alpha}\left(2+\left|\Omega\left(y^{\prime}\right)\right|\right) d \sigma\left(y^{\prime}\right)<\infty
$$

Denote by $L(\log L)\left(S^{n-1}\right)$ for $L(\log L)^{1}\left(S^{n-1}\right)$. A well-known fact is $L(\log L)\left(S^{n-1}\right) \subset H^{1}\left(S^{n-1}\right)$.

Next, we turn to the block space $B_{q}^{(0, v)}\left(S^{n-1}\right)$. A $q$-block on $S^{n-1}$ is an $L^{q}\left(S^{n-1}\right)(1<q \leq \infty)$ function $b$ which satisfies

(i) $\operatorname{supp} b \subset I$;

(ii) $\|b\|_{q} \leq|I|^{-1 / q^{\prime}}$,

where $|I|=\sigma(I)$, and $I=B\left(x_{0}^{\prime}, \theta_{0}\right) \cap S^{n-1}$ is a cap on $S^{n-1}$ for some $x_{0}^{\prime} \in S^{n-1}$ and $\theta_{0} \in(0,1]$. For $1<q \leq \infty$ and $v>-1$, the block space $B_{q}^{(0, v)}\left(S^{n-1}\right)$ is defined by

$$
B_{q}^{(0, v)}\left(S^{n-1}\right)=\left\{\Omega \in L^{1}\left(S^{n-1}\right) ; \Omega=\sum_{j=1}^{\infty} \lambda_{j} b_{j}, M_{q}^{(0, v)}\left(\left\{\lambda_{j}\right\}\right)<\infty\right\},
$$


where $\lambda_{j} \in \mathbb{C}$ and $b_{j}$ is a $q$-block supported on a cap $I_{j}$ on $S^{n-1}$, and

$$
M_{q}^{(0, v)}\left(\left\{\lambda_{j}\right\}\right)=\sum_{j=1}^{\infty}\left|\lambda_{j}\right|\left\{1+\log ^{(v+1)}\left(\left|I_{j}\right|^{-1}\right)\right\}
$$

For $\Omega \in B_{q}^{(0, v)}\left(S^{n-1}\right)$, denote

$$
\|\Omega\|_{B_{q}^{(0, v)}\left(S^{n-1}\right)}=\inf \left\{M_{q}^{(0, v)}\left(\left\{\lambda_{j}\right\}\right) ; \Omega=\sum_{j=1}^{\infty} \lambda_{j} b_{j}, b_{j} \text { is a } q \text {-block }\right\} .
$$

Then $\|\cdot\|_{B_{q}^{(0, v)}\left(S^{n-1}\right)}$ is a norm on the space $B_{q}^{(0, v)}\left(S^{n-1}\right)$, and $\left(B_{q}^{(0, v)}\left(S^{n-1}\right),\|\cdot\|_{B_{q}^{(0, v)}\left(S^{n-1}\right)}\right)$ is a Banach space.

Historically, the block spaces in $\mathbb{R}^{n}$ originated in the work of Taibleson and Weiss on the convergence of the Fourier series in connection with the developments of the real Hardy spaces. The block spaces on $S^{n-1}$ were introduced by Jiang and Lu [16] in studying the homogeneous singular integral operators. For further information about the theory of spaces generated by blocks and its applications to harmonic analysis, see the book [17] and survey article [18]. The following inclusion relations are known:
(a) $B_{q}^{\left(0, v_{1}\right)}\left(S^{n-1}\right) \subset B_{q}^{\left(0, v_{2}\right)}\left(S^{n-1}\right)$ if $v_{1}>v_{2}>-1$;
(b) $B_{q_{1}}^{(0, v)}\left(S^{n-1}\right) \subset B_{q_{2}}^{(0, v)}\left(S^{n-1}\right)$ if $1<q_{2}<q_{1}$ for any $v>-1$;
(c) $\bigcup_{p>1} L^{p}\left(S^{n-1}\right) \subset B_{q}^{(0, v)}\left(S^{n-1}\right)$ for any $q>1, v>-1$;
(d) $\bigcup_{q>1} B_{q}^{(0, v)}\left(S^{n-1}\right) \not \subset \bigcup_{q>1} L^{q}\left(S^{n-1}\right) \quad$ for any $v>-1$;
(e) $B_{q}^{(0, v)}\left(S^{n-1}\right) \subset H^{1}\left(S^{n-1}\right)+L(\log L)^{1+v}\left(S^{n-1}\right) \quad$ for any $q>1, v>-1$;
(f) $\bigcup_{q>1} B_{q}^{(0,0)}\left(S^{n-1}\right) \subset H^{1}\left(S^{n-1}\right)$.

The following theorem shows that if $\Omega$ belongs to $L \log L\left(S^{n-1}\right)$ or block spaces, then we can get better results than Theorem 1.1.

Theorem 1.2 Let $\phi$ be a positive increasing function on $(0, \infty)$ satisfying the same condition as in Theorem 1.1. Let $h \in \Delta_{\gamma}$ for some $1<\gamma \leq \infty$, and $\Omega \in L^{1}\left(S^{n-1}\right)$ satisfy the cancelation condition (1.1). Then if $\Omega \in L(\log L)\left(S^{n-1}\right) \cup\left(\bigcup_{1<q<\infty} B_{q}^{(0,0)}\left(S^{n-1}\right)\right)$, then

(i) $T_{\Omega, h, \phi}$ is bounded on $\dot{F}_{p, q}^{\alpha}\left(\mathbb{R}^{n}\right)$ for $\alpha \in \mathbb{R}$ and $p, q$ with $\left(\frac{1}{p}, \frac{1}{q}\right)$ belonging to the interior of the octagon $Q_{1} Q_{2} R_{2} P_{3} Q_{3} Q_{4} R_{4} P_{6}$ (hexagon $Q_{1} Q_{2} P_{3} Q_{3} Q_{4} P_{6}$ in the case $1<\gamma \leq 2$ ), where $Q_{1}=(0,0), Q_{2}=\left(\frac{1}{\gamma^{\prime}}, 0\right), Q_{3}=(1,1), Q_{4}=\left(\frac{1}{\gamma}, 1\right), P_{3}=\left(\frac{1}{2}+\frac{1}{\max \left\{2, \gamma^{\prime}\right\}}, \frac{1}{2}\right)$, $P_{6}=\left(\frac{1}{2} 1 \frac{1}{\max \left\{2, \gamma^{\prime}\right\}}, \frac{1}{2}\right), R_{2}=\left(1-\frac{1}{2 \gamma}, \frac{1}{2 \gamma}\right)$, and $R_{4}=\left(\frac{1}{2 \gamma}, 1-\frac{1}{2 \gamma}\right)$;

(ii) $T_{\Omega, h, \phi}$ is bounded on $\dot{B}_{p, q}^{\alpha}\left(\mathbb{R}^{n}\right)$ for $\alpha \in \mathbb{R}$ and $1<p, q<\infty$.

See Figures 3 and 4 for the conclusion of Theorem 1.2 for the cases $1<\gamma<2$ and $2 \leq$ $\gamma<\infty$, respectively.

As a corresponding result to Theorem $\mathrm{C}$, we have the following theorem. 

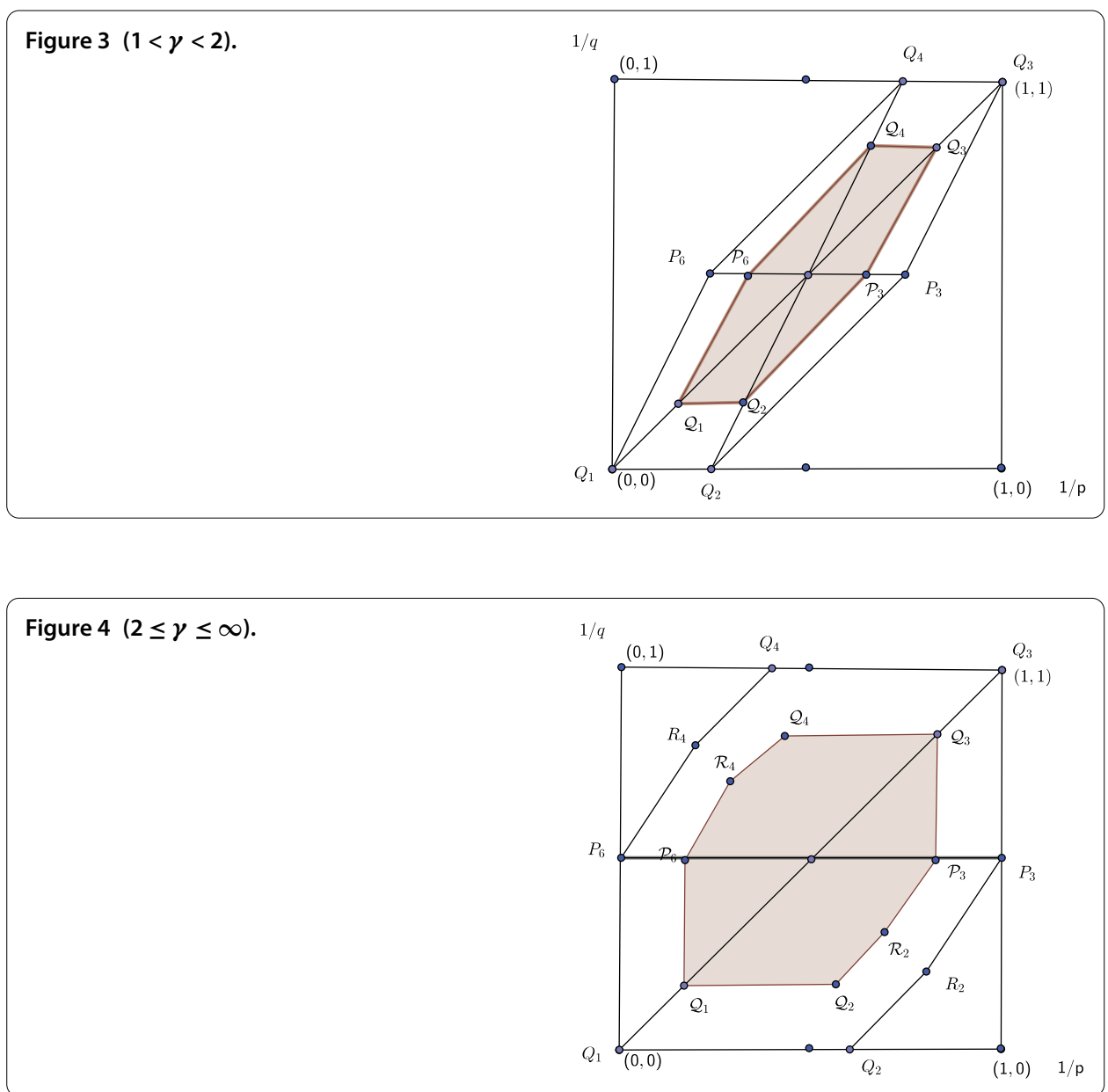

Theorem 1.3 Let $\phi$ be a positive increasing function on $(0, \infty)$ satisfying the same condition as in Theorem 1.1. Let $h \in \Delta_{\gamma}$ for some $1<\gamma \leq \infty$. Suppose $\Omega \in W_{\mathcal{F}}=W_{\mathcal{F}_{\beta}}\left(S^{n-1}\right)$ for some $\beta>\max \left(\gamma^{\prime}, 2\right)$, and it satisfies the cancelation condition (1.1). Then

(i) the singular integral operator $T_{\Omega, h, \phi}$ is bounded on $\dot{F}_{p, q}^{\alpha}\left(\mathbb{R}^{n}\right)$, if $\alpha \in \mathbb{R}$ and $\left(\frac{1}{p}, \frac{1}{q}\right)$ belongs to the interior of the octagon $\mathcal{Q}_{1} \mathcal{Q}_{2} \mathcal{R}_{2} \mathcal{P}_{3} \mathcal{Q}_{3} \mathcal{Q}_{4} \mathcal{R}_{4} \mathcal{P}_{6}$ (hexagon $\mathcal{Q}_{1} \mathcal{Q}_{2} \mathcal{P}_{3} \mathcal{Q}_{3} \mathcal{Q}_{4} \mathcal{P}_{6}$ in the case $\left.1<\gamma \leq 2\right)$, where $\mathcal{Q}_{1}=\left(\frac{\max \left(\gamma^{\prime}, 2\right)}{2 \beta}, \frac{\max \left(\gamma^{\prime}, 2\right)}{2 \beta}\right)$, $\mathcal{Q}_{2}=\left(\frac{1}{\gamma^{\prime}}+\frac{\max \left(\gamma^{\prime}, 2\right)}{\beta}\left(\frac{1}{2}-\frac{1}{\gamma^{\prime}}\right), \frac{\max \left(\gamma^{\prime}, 2\right)}{2 \beta}\right), \mathcal{P}_{3}=\left(\frac{1}{2}+\frac{1}{\max \left(\gamma^{\prime}, 2\right)}-\frac{1}{\beta}, \frac{1}{2}\right)$, $\mathcal{Q}_{3}=\left(1-\frac{\max \left(\gamma^{\prime}, 2\right)}{2 \beta}, 1-\frac{\max \left(\gamma^{\prime}, 2\right)}{2 \beta}\right), \mathcal{Q}_{4}=\left(\frac{1}{\gamma}-\frac{\max \left(\gamma^{\prime}, 2\right)}{\beta}\left(\frac{1}{\gamma}-\frac{1}{2}\right), 1-\frac{\max \left(\gamma^{\prime}, 2\right)}{2 \beta}\right)$, $\mathcal{P}_{6}=\left(\frac{1}{2}-\frac{1}{\max \left(\gamma^{\prime}, 2\right)}+\frac{1}{\beta}, \frac{1}{2}\right), \mathcal{R}_{2}=\left(1-\frac{1}{2 \gamma}-\frac{\max \left(\gamma^{\prime}, 2\right)}{2 \beta \gamma^{\prime}}, \frac{1}{2 \gamma}+\frac{\max \left(\gamma^{\prime}, 2\right)}{2 \beta \gamma^{\prime}}\right)$, and $\mathcal{R}_{4}=\left(\frac{1}{2 \gamma}+\frac{\max \left(\gamma^{\prime}, 2\right)}{2 \beta \gamma^{\prime}}, 1-\frac{1}{2 \gamma}-\frac{\max \left(\gamma^{\prime}, 2\right)}{2 \beta \gamma^{\prime}}\right)$;

(ii) $T_{\Omega, h, \phi}$ is bounded on $\dot{B}_{p, q}^{\alpha}\left(\mathbb{R}^{n}\right)$, if $\alpha \in \mathbb{R}, \frac{\max \left(\gamma^{\prime}, 2\right)}{2 \beta}<p<1-\frac{\max \left(\gamma^{\prime}, 2\right)}{2 \beta}$ and $1<q<\infty$.

This improves Theorem C sufficiently. See Figures 3 and 4 for the conclusion (i) of Theorem 1.3.

The proofs of Theorems 1.1 and 1.3 will be given in Sections 2 and 3, respectively, and the proof of Theorem 1.2 will be given in Section 4. The letter $C$ will denote a positive constant that may vary at each occurrence but is independent of the essential variables. 


\section{Proof of Theorem 1.1}

\subsection{Some lemmas}

In [19], the following atom-decomposition of $H^{1}\left(S^{n-1}\right)$ was given. If $\Omega \in H^{1}\left(S^{n-1}\right)$ satisfying (1.1), then

$$
\Omega=\sum_{j=1}^{\infty} \lambda_{j} a_{j},
$$

where $\sum_{j=1}^{\infty}\left|\lambda_{j}\right| \leq C\|\Omega\|_{H^{1}\left(S^{n-1}\right)}$ and each $a_{j}$ is a regular $H^{1}\left(S^{n-1}\right)$ atom. A function $a$ on $S^{n-1}$ is called regular $\infty$-atom in $H^{1}\left(S^{n-1}\right)$ if there exist $\zeta \in S^{n-1}$ and $\rho \in(0,2]$ such that

(i) $\operatorname{supp}(a) \subset S^{n-1} \cap B(\zeta, \rho)$, where $B(\zeta, \rho)=\left\{y \in R^{n}:|y-\zeta|<\rho\right\}$;

(ii) $\|a\|_{L^{\infty}} \leq \rho^{-n+1}$;

(iii) $\int_{S^{n-1}} a(y) d \sigma(y)=0$.

Let $a$ be a regular $\infty$-atom. When $n \geq 3$, set

$$
E_{a}\left(s, \xi^{\prime}\right)=\left(1-s^{2}\right)^{\frac{n-3}{2}} \chi_{(-1,1)}(s) \int_{S^{n-2}} a\left(s, \sqrt{1-s^{2}} \tilde{y}\right) d \sigma(\tilde{y})
$$

and when $n=2$, set

$$
e_{a}\left(s, \xi^{\prime}\right)=\frac{1}{\sqrt{1-s^{2}}} \chi_{(-1,1)}(s)\left[a\left(s, \sqrt{1-s^{2}}\right)+a\left(s,-\sqrt{1-s^{2}}\right)\right] .
$$

Next we prepare two lemmas, whose proofs can be found in Fan and Pan [4].

Lemma 2.1 Let $\Omega$ be a regular $\infty$-atom in $H^{1}\left(S^{n-1}\right)(n \geq 3)$. Then there exists a constant $c>0$, independent of $\Omega$, such that $c E_{\Omega}\left(s, \xi^{\prime}\right)$ is an $\infty$-atom in $H^{1}(\mathbb{R})$. That is, $c E_{\Omega}\left(s, \xi^{\prime}\right)$ satisfies

$$
\begin{aligned}
& \left\|c E_{\Omega}\right\|_{L^{\infty}} \leq \frac{1}{4 r\left(\xi^{\prime}\right)}, \quad \operatorname{supp} E_{\Omega} \subset\left(\xi_{1}^{\prime}-2 r\left(\xi^{\prime}\right), \xi_{1}^{\prime}+2 r\left(\xi^{\prime}\right)\right) \text { and } \\
& \int_{\mathbb{R}} E_{\Omega}\left(s, \xi^{\prime}\right) d s=0,
\end{aligned}
$$

where $r\left(\xi^{\prime}\right)=|\xi|^{-1}\left|A_{\tau} \xi\right|$ and $A_{\tau}(\xi)=\left(\tau^{2} \xi_{1}, \tau \xi_{2}, \ldots, \tau \xi_{n}\right)$

Lemma 2.2 Let $\Omega$ be a regular $\infty$-atom in $H^{1}\left(S^{1}\right)$. Then, for $1<q<2$, there exists a constant $c>0$, independent of $\Omega$, such that $c e_{\Omega}\left(s, \xi^{\prime}\right)$ is a q-atom in $H^{1}(\mathbb{R})$, the center of whose support is $\xi_{1}^{\prime}$ and the radius $r\left(\xi^{\prime}\right)=|\xi|^{-1}\left(\tau^{4} \xi_{1}^{2}+\tau^{2} \xi_{2}^{2}\right)^{1 / 2}$.

For $\Omega \in L^{1}\left(S^{n-1}\right), h \in \Delta_{\gamma}$ for some $1<\gamma \leq \infty$, and a suitable function $\phi$ on $\mathbb{R}_{+}$, we define the maximal functions $M_{\Omega, h, \phi}$ by

$$
M_{\Omega, h, \phi} f(x)=\sup _{k \in \mathbb{Z}} \frac{1}{2^{k n}} \int_{2^{k-1}<|y| \leq 2^{k}}\left|\Omega\left(y^{\prime}\right) h(|y|) f\left(x-\phi(|y|) y^{\prime}\right)\right| d y .
$$

Let $\phi$ be a positive increasing function on $(0, \infty)$ satisfying $\phi(2 t) \leq c_{1} \phi(t)(t>0)$ for some $c_{1}>1$, and $\varphi(t)=\phi(t) /\left(t \phi^{\prime}(t)\right) \in L^{\infty}(0, \infty)$. Then, as in the proof of Lemma 2.3 in [20], 
p.246, we have

$$
\begin{aligned}
M_{\Omega, h, \phi} f(x) \leq & \|h\|_{\Delta_{\gamma}}\left(\|\Omega\|_{L^{1}\left(S^{n-1}\right)}\right)^{\frac{1}{\gamma}} \\
& \times\left(\int_{S^{n-1}}\left|\Omega\left(y^{\prime}\right)\right| M_{y^{\prime}}\left(|f|^{\gamma^{\prime}}\right)(x) d \sigma\left(y^{\prime}\right)\right)^{\frac{1}{\gamma^{\prime}}},
\end{aligned}
$$

where $M_{y^{\prime}} g$ is the directional Hardy-Littlewood maximal function of $g$ defined by

$$
M_{y^{\prime}} g(x)=\sup _{r>0} \frac{1}{2 r} \int_{|t|<r}\left|g\left(x-t y^{\prime}\right)\right| d t .
$$

For this directional maximal function $M_{y^{\prime}}$, we know that for $1<p, q<\infty$,

$$
\begin{aligned}
& \left(\int_{\mathbb{R}^{n}}\left[\left(\sum_{j \in \mathbb{Z}}\left(M_{y^{\prime}}\left(f_{j}\right)(x)\right)^{q}\right)^{\frac{1}{q}}\right]^{p} d x\right)^{\frac{1}{p}} \\
& \leq C_{p, q}\left(\int_{\mathbb{R}^{n}}\left[\left(\sum_{j \in \mathbb{Z}}\left|f_{j}(x)\right|^{q}\right)^{\frac{1}{q}}\right]^{p} d x\right)^{\frac{1}{p}} .
\end{aligned}
$$

This is just (2.7) in the proof of Lemma 2.3 of [8], p.496. From (2.6) and (2.7), we get the following lemma.

Lemma 2.3 Let $\phi$ be a positive increasing function on $(0, \infty)$ satisfying $\phi(2 t) \leq c_{1} \phi(t)(t>$ 0) for some $c_{1}>1$, and $\varphi(t)=\phi(t) /\left(t \phi^{\prime}(t)\right) \in L^{\infty}(0, \infty)$. Let $h \in \Delta_{\gamma}$ for some $1<\gamma \leq \infty$. For $\gamma^{\prime}<p, q<\infty$, we have

$$
\left\|\left(\sum_{j \in \mathbb{Z}}\left|M_{\Omega, h, \phi} f_{j}\right|^{q}\right)^{\frac{1}{q}}\right\|_{L^{p}\left(\mathbb{R}^{n)}\right.} \leq C\left\|\left(\sum_{j \in \mathbb{Z}}\left|f_{j}\right|^{q}\right)^{\frac{1}{q}}\right\|_{L^{p}\left(\mathbb{R}^{n}\right)} .
$$

Proof Let $\left\{g_{j}\right\}_{j \in \mathbb{Z}}$ be a sequence of functions satisfying $\left\|\left(\sum_{j \in \mathbb{Z}}\left|g_{j}\right|^{q^{\prime}}\right)^{1 / q^{\prime}}\right\|_{L^{p^{\prime}}\left(\mathbb{R}^{n}\right)} \leq 1$. Then, noting $p, q>\gamma^{\prime}$ and using (2.6), the duality, and Minkowski's inequality, we see that

$$
\begin{aligned}
& \left|\int_{\mathbb{R}^{n}} \sum_{j \in \mathbb{Z}} M_{\Omega, h, \phi} f_{j}(x) g_{j}(x) d x\right| \\
& \leq C \int_{\mathbb{R}^{n}} \sum_{j \in \mathbb{Z}}\left(\int_{S^{n-1}}\left|\Omega\left(y^{\prime}\right)\right| M_{y^{\prime}}\left(\left|f_{j}\right|^{\gamma^{\prime}}\right)(x) d \sigma\left(y^{\prime}\right)\right)^{\frac{1}{\gamma^{\prime}}}\left|g_{j}(x)\right| d x \\
& \leq C\left(\int_{\mathbb{R}^{n}}\left(\sum_{j \in \mathbb{Z}}\left(\int_{S^{n-1}}\left|\Omega\left(y^{\prime}\right)\right| M_{y^{\prime}}\left(\left|f_{j}\right|^{\gamma^{\prime}}\right)(x) d \sigma\left(y^{\prime}\right)\right)^{\frac{q}{\gamma^{\prime}}}\right)^{\frac{p}{q}} d x\right)^{\frac{1}{p}} \\
& \quad \times\left\|\left(\sum_{j \in \mathbb{Z}}\left|g_{j}(x)\right|^{q^{\prime}}\right)^{\frac{1}{q^{\prime}}}\right\| \|_{L^{p^{\prime}}\left(\mathbb{R}^{n}\right)} \\
& \leq C\left\{\int_{S^{n-1}}\left|\Omega\left(y^{\prime}\right)\right|\left(\int_{\mathbb{R}^{n}}\left[\left(\sum_{j \in \mathbb{Z}}\left(M_{y^{\prime}}\left(\left|f_{j}\right|^{\gamma^{\prime}}\right)(x)\right)^{\frac{q}{\gamma^{\prime}}}\right)^{\frac{\gamma^{\prime}}{q}}\right]^{\frac{p}{\gamma^{\prime}}} d x\right)^{\frac{\gamma^{\prime}}{p}} d \sigma\left(y^{\prime}\right)\right\}^{\frac{1}{\gamma^{\prime}}} .
\end{aligned}
$$


Hence by (2.7) we have

$$
\begin{aligned}
\left|\int_{\mathbb{R}^{n}} \sum_{j \in \mathbb{Z}} M_{\Omega, h, \phi} f_{j}(x) g_{j}(x) d x\right| & \leq C\left\{\int_{S^{n-1}}\left|\Omega\left(y^{\prime}\right)\right|\left(\int_{\mathbb{R}^{n}}\left(\sum_{j \in \mathbb{Z}}\left|f_{j}(x)\right|^{q}\right)^{\frac{p}{q}} d x\right)^{\frac{\gamma^{\prime}}{p}} d \sigma\left(y^{\prime}\right)\right\}^{\frac{1}{\gamma^{\prime}}} \\
& \leq C\left\|\left(\sum_{j \in \mathbb{Z}}\left|f_{j}\right|^{q}\right)^{\frac{1}{q}}\right\|_{L^{p}\left(\mathbb{R}^{n}\right)},
\end{aligned}
$$

which implies our (2.8).

Now, for $\Omega \in L^{1}\left(S^{n-1}\right)$, we define the measures $\sigma_{\Omega, h, \phi, k}$ on $\mathbb{R}^{n}$ and the maximal operator $\sigma_{\Omega, h, \phi}^{*} f(x)$ by

$$
\begin{aligned}
& \int_{\mathbb{R}^{n}} f(x) d \sigma_{\Omega, h, \phi, k}(x)=\int_{\mathbb{R}^{n}} f\left(\phi(|x|) x^{\prime}\right) \frac{\Omega\left(x^{\prime}\right) h(|x|)}{|x|^{n}} \chi_{2^{k-1}<|x| \leq 2^{k}}(x) d x, \\
& \sigma_{\Omega, h, \phi}^{*} f(x)=\sup _{k \in \mathbb{Z}}|| \sigma_{\Omega, h, \phi, k}|* f(x)|,
\end{aligned}
$$

where $\left|\sigma_{\Omega, h, \phi, k}\right|$ is defined in the same way as $\sigma_{\Omega, h, \phi, k}$, but with $\Omega$ replaced by $|\Omega|$ and $h$ by $|h|$.

Then we have the following lemma.

Lemma 2.4 Let $\phi$ be a positive increasing function on $(0, \infty)$ satisfying $\phi(2 t) \leq c_{1} \phi(t)(t>$ 0) for some $c_{1}>1$, and $\varphi(t)=\phi(t) /\left(t \phi^{\prime}(t)\right) \in L^{\infty}(0, \infty)$. Let $h \in \Delta_{\gamma}$ for some $1<\gamma \leq \infty$, $\Omega \in L^{1}\left(S^{n-1}\right)$. Then:

(i) If $\left(\frac{1}{p}, \frac{1}{q}\right)$ belongs to the interior of the octagon $P_{1} P_{2} R_{2} P_{3} P_{4} P_{5} R_{4} P_{6}$, there exists $C>0$ such that

$$
\begin{aligned}
& \left\|\left(\sum_{j \in \mathbb{Z}}\left(\sum_{k \in \mathbb{Z}}\left|\sigma_{\Omega, h, \phi, k} * g_{k, j}\right|^{2}\right)^{\frac{q}{2}}\right)^{\frac{1}{q}}\right\|_{L^{p}\left(\mathbb{R}^{n}\right)} \\
& \leq C\|h\|_{\Delta_{\gamma}}\|\Omega\|_{L^{1}\left(S^{n-1}\right)}\left\|\left(\sum_{j \in \mathbb{Z}}\left(\sum_{k \in \mathbb{Z}}\left|g_{k, j}\right|^{2}\right)^{\frac{q}{2}}\right)^{\frac{1}{q}}\right\|_{L^{p}\left(\mathbb{R}^{n}\right)},
\end{aligned}
$$

where $P_{1}=\left(\frac{1}{2}-\frac{1}{\max \left\{2, \gamma^{\prime}\right\}}, \frac{1}{2}-\frac{1}{\max \left\{2, \gamma^{\prime}\right\}}\right), P_{2}=\left(\frac{1}{2}, \frac{1}{2}-\frac{1}{\max \left\{2, \gamma^{\prime}\right\}}\right), P_{3}=\left(\frac{1}{2}+\frac{1}{\max \left\{2, \gamma^{\prime}\right\}}, \frac{1}{2}\right)$, $P_{4}=\left(\frac{1}{2}+\frac{1}{\max \left\{2, \gamma^{\prime}\right\}}, \frac{1}{2}+\frac{1}{\max \left\{2, \gamma^{\prime}\right\}}\right), P_{5}=\left(\frac{1}{2}, \frac{1}{2}+\frac{1}{\max \left\{2, \gamma^{\prime}\right\}}\right), P_{6}=\left(\frac{1}{2}-\frac{1}{\max \left\{2, \gamma^{\prime}\right\}}, \frac{1}{2}\right)$, $R_{2}=\left(1-\frac{1}{2 \gamma}, \frac{1}{2 \gamma}\right)$, and $R_{4}=\left(\frac{1}{2 \gamma}, 1-\frac{1}{2 \gamma}\right)$.

(Note that if $1<\gamma \leq 2$, the octagon $P_{1} P_{2} R_{2} P_{3} P_{4} P_{5} R_{4} P_{6}$ reduces to the hexagon $P_{1} P_{2} P_{3} P_{4} P_{5} P_{6}$.)

(ii) If $\left(\frac{1}{p}, \frac{1}{q}\right)$ belongs to the interior of $Q_{1} Q_{2} Q_{3} Q_{4}$, there exists $C>0$ such that

$$
\begin{aligned}
& \left\|\left(\sum_{j \in \mathbb{Z}} \sum_{k \in \mathbb{Z}}\left|\sigma_{\Omega, h, \phi, k} * g_{k, j}\right|^{q}\right)^{\frac{1}{q}}\right\|_{L^{p}\left(\mathbb{R}^{n}\right)} \\
& \leq C\|h\|_{\Delta_{\gamma}}\|\Omega\|_{L^{1}\left(S^{n-1}\right)}\left\|\left(\sum_{j \in \mathbb{Z}} \sum_{k \in \mathbb{Z}}\left|g_{k, j}\right|^{q}\right)^{\frac{1}{q}}\right\|_{L^{p}\left(\mathbb{R}^{n}\right)},
\end{aligned}
$$

where $Q_{1}=(0,0), Q_{2}=\left(\frac{1}{\gamma^{\prime}}, 0\right), Q_{3}=(1,1)$, and $Q_{4}=\left(\frac{1}{\gamma}, 1\right)$. 
Proof (a) Let $1<\gamma \leq \infty$. Since

$$
\sup _{k \in \mathbb{Z}}\left|\sigma_{\Omega, h, \phi, k} * g_{k, j}\right| \leq \sup _{k \in \mathbb{Z}}\left|\sigma_{\Omega, h, \phi, k}\right| * \sup _{\ell \in \mathbb{Z}}\left|g_{\ell, j}\right| \leq M_{\Omega, h, \phi}\left(\sup _{\ell \in \mathbb{Z}}\left|g_{\ell, j}\right|\right)
$$

we get using Lemma 2.3

$$
\begin{aligned}
\left\|\left(\sum_{j \in \mathbb{Z}}\left(\sup _{k \in \mathbb{Z}}\left|\sigma_{\Omega, h, \phi, k} * g_{k, j}\right|\right)^{q}\right)^{\frac{1}{q}}\right\|_{L^{p}\left(\mathbb{R}^{n}\right)} & \leq\left\|\left(\sum_{j \in \mathbb{Z}}\left(M_{\Omega, h, \phi}\left(\sup _{k \in \mathbb{Z}}\left|g_{k, j}\right|\right)\right)^{q}\right)^{\frac{1}{q}}\right\|_{L^{p}\left(\mathbb{R}^{n}\right)} \\
& \leq C\left\|\left(\sum_{j \in \mathbb{Z}}\left(\sup _{k \in \mathbb{Z}}\left|g_{k, j}\right|\right)^{q}\right)^{\frac{1}{q}}\right\|_{L^{p}\left(\mathbb{R}^{n}\right)} .
\end{aligned}
$$

On the other hand, there exists $\left\{h_{j}\right\} \in L^{p^{\prime}}\left(\ell^{q^{\prime}}\right)$ with $\left\|\left\{h_{j}\right\}\right\|_{L^{p^{\prime}}\left(\ell q^{\prime}\right)}=1$ such that

$$
\begin{aligned}
& \left\|\left(\sum_{j \in \mathbb{Z}}\left(\sum_{k \in \mathbb{Z}}\left|\sigma_{\Omega, h, \phi, k} * g_{k, j}\right|\right)^{q}\right)^{\frac{1}{q}}\right\|_{L^{p}\left(\mathbb{R}^{n}\right)} \\
& \quad=\sum_{j \in \mathbb{Z}} \int_{\mathbb{R}^{n}} \sum_{k \in \mathbb{Z}}\left|\sigma_{\Omega, h, \phi, k} * g_{k, j}(x)\right| h_{j}(x) d x \\
& \quad \leq \sum_{j \in \mathbb{Z}} \int_{\mathbb{R}^{n}} \sum_{k \in \mathbb{Z}}\left|g_{k, j}(x)\right|\left|\tilde{\sigma}_{\Omega, h, \phi, k}\right| * h_{j}(x) d x \\
& \quad \leq \sum_{j \in \mathbb{Z}} \int_{\mathbb{R}^{n}} \sum_{k \in \mathbb{Z}}\left|g_{k, j}(x)\right| M_{\tilde{\Omega}, h, \phi} h_{j}(x) d x \\
& \quad \leq C\left\|\left(\sum_{j \in \mathbb{Z}}\left(\sum_{k \in \mathbb{Z}}\left|g_{k, j}\right|\right)^{q}\right)^{\frac{1}{q}}\right\|\left\|_{L^{p}\left(\mathbb{R}^{n}\right)}\right\|\left(\sum_{j \in \mathbb{Z}}\left(M_{\tilde{\Omega}, h, \phi} h_{j}(x)\right)^{q^{\prime}}\right)^{\frac{1}{q^{\prime}}} \|_{L^{p^{\prime}}\left(\mathbb{R}^{n}\right)},
\end{aligned}
$$

where $\tilde{\Omega}\left(y^{\prime}\right)=\Omega\left(-y^{\prime}\right)$. So by Lemma 2.3 we obtain for $\gamma^{\prime}<p^{\prime}, q^{\prime}<\infty$, i.e., $1<p, q<\gamma$,

$$
\begin{aligned}
& \left\|\left(\sum_{j \in \mathbb{Z}}\left(\sum_{k \in \mathbb{Z}}\left|\sigma_{\Omega, h, \phi, k} * g_{k, j}\right|\right)^{q}\right)^{\frac{1}{q}}\right\|_{L^{p}\left(\mathbb{R}^{n}\right)} \\
& \leq C\left\|\left(\sum_{j \in \mathbb{Z}}\left(\sum_{k \in \mathbb{Z}}\left|g_{k, j}\right|\right)^{q}\right)^{\frac{1}{q}}\right\|_{L^{p}\left(\mathbb{R}^{n}\right)}\left\|\left(\sum_{j \in \mathbb{Z}}\left(\left|h_{j}(x)\right|\right)^{q^{\prime}}\right)^{\frac{1}{q^{\prime}}}\right\|_{L^{p^{\prime}}\left(\mathbb{R}^{n}\right)} \\
& \leq C\left\|\left(\sum_{j \in \mathbb{Z}}\left(\sum_{k \in \mathbb{Z}}\left|g_{k, j}\right|\right)^{q}\right)^{\frac{1}{q}}\right\|_{L^{p}\left(\mathbb{R}^{n}\right)} .
\end{aligned}
$$

Now, let $R_{1}=\left(\frac{1}{2 \gamma}, \frac{1}{2 \gamma}\right), R_{2}=\left(1-\frac{1}{2 \gamma}, \frac{1}{2 \gamma}\right), R_{3}=\left(1-\frac{1}{2 \gamma}, 1-\frac{1}{2 \gamma}\right)$, and $R_{4}=\left(\frac{1}{2 \gamma}, 1-\frac{1}{2 \gamma}\right)$. Then, if $\left(\frac{1}{p}, \frac{1}{q}\right)$ belongs to the interior of the square $R_{1} R_{2} R_{3} R_{4}$, there are two points $\left(\frac{1}{p_{1}}, \frac{1}{q_{1}}\right)$ and $\left(\frac{1}{p_{2}}, \frac{1}{q_{2}}\right)$ such that

$$
\begin{array}{ll}
\frac{1}{p}=\frac{1}{2} \frac{1}{p_{1}}+\frac{1}{2} \frac{1}{p_{2}}, & \frac{1}{q}=\frac{1}{2} \frac{1}{q_{1}}+\frac{1}{2} \frac{1}{q_{2}}, \\
1<p_{1}, q_{1}<\gamma & \text { and } \quad \gamma^{\prime}<p_{2}, q_{2}<\infty .
\end{array}
$$


Hence, interpolating (2.13) with (2.14), we obtain (2.11) if $\left(\frac{1}{p}, \frac{1}{q}\right)$ belongs to the interior of the square $R_{1} R_{2} R_{3} R_{4}$.

(b) Let $1<\gamma<2$. Using the Cauchy-Schwarz inequality, we get

$$
\begin{aligned}
\left|\sigma_{\Omega, h, \phi, k} * g_{k, j}(x)\right| \leq & \left(\int_{2^{k-1} \leq|y| \leq 2^{k}} \frac{\left|\Omega\left(y^{\prime}\right)\right||h(|y|)|^{\gamma}}{|y|^{n}} d y\right)^{\frac{1}{2}} \\
& \times\left(\int_{2^{k-1} \leq|y| \leq 2^{k}}\left|g_{k, j}\left(x-\phi(|y|) y^{\prime}\right)\right|^{2} \frac{\left|\Omega\left(y^{\prime}\right)\right||h(|y|)|^{2-\gamma}}{|y|^{n}} d y\right)^{\frac{1}{2}} \\
\leq & C\|h\|_{\Delta_{\gamma}}^{\frac{\gamma}{2}}\|\Omega\|_{L^{1}\left(S^{n-1}\right)}^{\frac{1}{2}}\left(\sigma_{|\Omega|,|h|^{2-\gamma}, \phi, k} *\left|g_{k, j}\right|^{2}\right)(x)^{\frac{1}{2}} .
\end{aligned}
$$

So, we have

$$
\begin{aligned}
& \left\|\left(\sum_{j \in \mathbb{Z}}\left(\sum_{k \in \mathbb{Z}}\left|\sigma_{\Omega, h, \phi, k} * g_{k, j}\right|^{2}\right)^{\frac{q}{2}}\right)^{\frac{1}{q}}\right\|_{L^{p}\left(\mathbb{R}^{n}\right)} \\
& \quad \leq C\|h\|_{\Delta_{\gamma}}^{\frac{\gamma}{2}}\|\Omega\|_{L^{1}\left(S^{n-1}\right)}^{\frac{1}{2}}\left\|\left(\sum_{j \in \mathbb{Z}}\left(\sum_{k \in \mathbb{Z}} \sigma_{|\Omega|,|h|^{2-\gamma}, \phi, k} *\left|g_{k, j}\right|^{2}\right)^{\frac{q}{2}}\right)^{\frac{1}{q}}\right\|_{L^{p}\left(\mathbb{R}^{n}\right)} .
\end{aligned}
$$

Hence, noting $|h|^{2-\gamma} \in \Delta_{\gamma /(2-\gamma)}$ and using (2.14) for $\gamma /(2-\gamma), p / 2$ and $q / 2$ in place of $\gamma, p$, $q$, respectively, we see that (2.11) holds provided $1<p / 2, q / 2<\gamma /(2-\gamma)$, i.e., $1 / 2-1 / \gamma^{\prime}<$ $1 / p, 1 / q<1 / 2$. By duality, it holds also provided $1 / 2<1 / p, 1 / q<1 / 2+1 / \gamma^{\prime}$. Interpolating these two cases, we see that (2.11) holds if $\left(\frac{1}{p}, \frac{1}{q}\right)$ belongs to the interior of the hexagon $P_{1} P_{2} P_{3} P_{4} P_{5} P_{6}$.

(c) Noting $\Delta_{\gamma} \subset \Delta_{2}$ for $\gamma>2$, and interpolating cases (a) and (b) above, we see that (2.11) holds if $\left(\frac{1}{p}, \frac{1}{q}\right)$ belongs to the interior of the octagon $P_{1} P_{2} R_{2} P_{3} P_{4} P_{5} R_{4} P_{6}$. This completes the proof of Lemma 2.4(i).

(d) We shall prove Lemma 2.4(ii). If $\left(\frac{1}{p}, \frac{1}{q}\right)$ belongs to the interior of the parallelogram $Q_{1} Q_{2} Q_{3} Q_{4}$, there are two points $\left(\frac{1}{p_{1}}, \frac{1}{q_{1}}\right)$ and $\left(\frac{1}{p_{2}}, \frac{1}{q_{2}}\right)$ such that

$$
\begin{aligned}
& \frac{1}{p}=\left(1-\frac{1}{q}\right) \frac{1}{p_{1}}+\frac{1}{q} \frac{1}{p_{2}}, \quad \frac{1}{q}=\left(1-\frac{1}{q}\right) \frac{1}{q_{1}}+\frac{1}{q} \frac{1}{q_{2}}, \\
& 1<p_{1}, q_{1}<\gamma \quad \text { and } \quad \gamma^{\prime}<p_{2}, q_{2}<\infty .
\end{aligned}
$$

Hence, interpolating (2.13) with (2.14), we obtain (2.12). Thus, we finished the proof of Lemma 2.4.

About the Fourier transform estimates of $\sigma_{\Omega, h, \phi, k}$ with $\Omega \in H^{1}\left(S^{n-1}\right)$, we have the following.

Lemma 2.5 Let $1<q \leq+\infty$ and $\Omega$ be a regular $\infty$-atom in $H^{1}\left(S^{n-1}\right)$ supported in $S^{n-1} \cap$ $B\left(\mathbf{e}_{1}, \tau\right)$, where $\mathbf{e}_{1}=(1,0, \ldots, 0)$. Let $\phi$ be a positive increasing function on $(0, \infty)$ satisfying $\varphi(t)=\phi(t) /\left(t \phi^{\prime}(t)\right) \in L^{\infty}(0, \infty)$, and $h \in \Delta_{\gamma}$ for some $1<\gamma \leq \infty$. Then there exist positive constants $C$ 's such that

$$
\begin{aligned}
& \left|\hat{\sigma}_{\Omega, h, \phi, k}(\xi)\right| \leq C\|h\|_{\Delta_{1}}\|\Omega\|_{L^{1}\left(S^{n-1}\right)}, \\
& \left|\hat{\sigma}_{\Omega, h, \phi, k}(\xi)\right| \leq C\|h\|_{\Delta_{1}} \phi\left(2^{k}\right)\left|A_{\tau}(\xi)\right|
\end{aligned}
$$


and

$$
\left|\hat{\sigma}_{\Omega, h, \phi, k}(\xi)\right| \leq \frac{C\|h\|_{\Delta_{\gamma}}}{\left(\phi\left(2^{k-1}\right)\left|A_{\tau}(\xi)\right|\right)^{1 / \max \left\{\gamma^{\prime}, 2\right\}}} .
$$

These are shown by using Lemmas 2.1 and 2.2 as in the proofs of Lemmas 3.3 and 3.4 in [20], pp.247-248. There these are stated for the case where a parameter $\rho$ of positive number arises, but one sees easily that these hold in our case $(\rho=0)$, too.

To show Theorem 1.1, we need a characterization of the Triebel-Lizorkin space in terms of lacunary sequences. Let $\left\{a_{j}\right\}_{j \in \mathbb{Z}}$ be a lacunary sequence with lacunarity $a>1$, i.e.,

$$
\frac{a_{j+1}}{a_{j}} \geq a \quad \text { for } j \in \mathbb{Z}
$$

Let $\eta$ be a radial function in $C^{\infty}\left(\mathbb{R}^{n}\right)$ satisfying $\chi_{|\xi| \leq 1}(\xi) \leq \eta(\xi) \leq \chi_{|\xi| \leq a}(\xi)$ and $\left|\partial^{\alpha} \eta(\xi)\right| \leq$ $c_{\alpha}(a-1)^{-|\alpha|}$ for $\xi \in \mathbb{R}^{n}$ and $\alpha \in \mathbb{Z}_{+}^{n}$. We define functions $\psi_{j}$ on $\mathbb{R}^{n}$ by

$$
\psi_{j}(\xi)=\eta\left(\frac{\xi}{a_{j+1}}\right)-\eta\left(\frac{\xi}{a_{j}}\right) \quad\left(\xi \in \mathbb{R}^{n}\right)
$$

Then observe that

$$
\psi_{j}(\xi)= \begin{cases}0, & 0 \leq|\xi| \leq a_{j},|\xi| \geq a a_{j+1} \\ 1, & a a_{j} \leq|\xi| \leq a_{j+1}\end{cases}
$$

and that

$$
\begin{aligned}
& \operatorname{supp} \psi_{j} \subset\left\{a_{j} \leq|\xi| \leq a a_{j+1}\right\}, \\
& \operatorname{supp} \psi_{j} \cap \operatorname{supp} \psi_{\ell}=\emptyset \quad \text { for }|j-\ell| \geq 2, \\
& \left|\xi^{\alpha} \partial^{\alpha} \psi_{j}(\xi)\right| \leq C_{\alpha} \quad \text { for } \alpha \in \mathbb{Z}_{+}^{n}, \\
& \sum_{j \in \mathbb{Z}} \psi_{j}(\xi)=1 \quad\left(\xi \in \mathbb{R}^{n} \backslash\{0\}\right) .
\end{aligned}
$$

Let $\Psi_{j}$ be defined on $\mathbb{R}^{n}$ by $\widehat{\Psi}_{j}(\xi)=\psi_{j}(\xi)$ for $\xi \in \mathbb{R}^{n}$, i.e., $\Psi_{j}(x)=a_{j+1}^{n} \check{\eta}\left(a_{j+1} x\right)-a_{j}^{n} \check{\eta}\left(a_{j} x\right)$.

Lemma 2.6 Define the multiplier $S_{j}$ by $S_{j} f=\Psi_{j} * f$. Then, for $1<p, q<\infty$, we have

$$
\left\|\left(\sum_{j \in \mathbb{Z}}\left(\sum_{k \in \mathbb{Z}}\left|S_{k} f_{j}\right|^{2}\right)^{q / 2}\right)^{1 / q}\right\|_{L^{p}\left(\mathbb{R}^{n}\right)} \leq C\left\|\left(\sum_{j \in \mathbb{Z}}\left|f_{j}\right|^{q}\right)^{1 / q}\right\|_{L^{p}\left(\mathbb{R}^{n}\right)},
$$

where $C$ is independent of $\left\{f_{j}\right\}_{j \in \mathbb{Z}}$.

This is a consequence of Proposition 4.6.4 in Grafakos [21]. For the sake of completeness, we will give a proof in the Appendix. From this lemma we have the following lemma with minor change of the proof of Lemma 2.2 in [8]. 
Lemma 2.7 Let $\psi_{j}$ be as in Lemma 2.6. Denote $A_{\tau}(\xi)=\left(\tau^{2} \xi_{1}, \tau \xi_{2}, \ldots, \tau \xi_{n}\right)$ for $\tau>0$ and $\xi \in \mathbb{R}^{n}$. Define the multiplier $S_{j, \tau}$ by $\widehat{S_{j, \tau} f}(\xi)=\psi\left(a_{k} A_{\tau}(\xi)\right) \hat{f}(\xi)$. Then, for $1<p, q<\infty$, we have

$$
\left\|\left(\sum_{j \in \mathbb{Z}}\left(\sum_{k \in \mathbb{Z}}\left|S_{k, \tau} f_{j}\right|^{2}\right)^{q / 2}\right)^{1 / q}\right\|_{L^{p}\left(\mathbb{R}^{n)}\right.} \leq C\left\|\left(\sum_{j \in \mathbb{Z}}\left|f_{j}\right|^{q}\right)^{1 / q}\right\|_{L^{p}\left(\mathbb{R}^{n}\right)},
$$

where $C$ is independent of $\left\{f_{j}\right\}_{j \in \mathbb{Z}}$.

We need one more lemma. If $\left\{a_{k}\right\}_{k \in \mathbb{Z}}$ satisfies furthermore $a_{k+1} / a_{k} \leq d$ for some $d \geq a$, we can characterize Triebel-Lizorkin spaces in terms of this lacunary sequence.

Denote by $\mathcal{P}$ the set of all polynomials in $\mathbb{R}^{n}$. Let $1<p, q<\infty$, and $\alpha \in \mathbb{R}$. For $f \in$

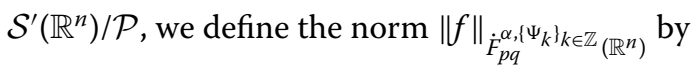

$$
\|f\|_{\dot{F}_{p q}^{\alpha,\left\{\Psi_{k}\right\}_{k \in \mathbb{Z}}\left(\mathbb{R}^{n}\right)}}=\left\|\left(\sum_{k \in \mathbb{Z}} a_{k}^{\alpha q}\left|\Psi_{k} * f\right|^{q}\right)^{1 / q}\right\|_{L^{p}\left(\mathbb{R}^{n)}\right.} .
$$

Lemma 2.8 Let $\alpha \in \mathbb{R}$ and $1<p, q<\infty$. Let $\left\{a_{k}\right\}_{k \in \mathbb{Z}}$ be a lacunary sequence of positive

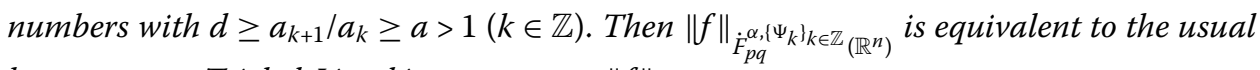
homogeneous Triebel-Lizorkin space norm $\|f\|_{\dot{F}_{p q}^{\alpha}\left(\mathbb{R}^{n}\right)}$.

This is stated in Proposition 1 in [22] for $\alpha \neq 0$, but the proof of this part works also for $\alpha=0$.

\subsection{Proof of Theorem 1.1}

We have only to show Theorem 1.1 in the case $\Omega$ is a regular atom with supp $\Omega \subset S^{n-1} \cap$ $B(\xi, \tau)$, where $B(\xi, \tau)=\left\{y \in \mathbb{R}^{n} ;|y-\xi|<\tau\right\}$. Using the definition of $\sigma_{\Omega, h, \phi, k}$, we see that

$$
T_{\Omega, h, \phi} f(x)=\text { p.v. } \int_{\mathbb{R}^{n}} \frac{h(|y|) \Omega\left(y^{\prime}\right)}{|y|^{n}} f\left(x-\phi(|y|) y^{\prime}\right) d y=\sum_{k \in \mathbb{Z}} \sigma_{\Omega, h, \phi, k} * f(x) .
$$

Let $a_{k}=1 / \phi\left(2^{-k}\right), k \in \mathbb{Z}$. Then as is known, $\left\{a_{k}\right\}_{k \in \mathbb{Z}}$ is a lacunary sequence with lacunarity


which follows from (1.9).

Let $\psi_{k} \in C_{c}^{\infty}\left(\mathbb{R}^{n}\right)$ be radial functions defined by (2.19). Set $\psi_{k, \tau}(\xi)=\psi_{k}\left(A_{\tau}(\xi)\right)$ and $\widehat{S_{k, \tau} f}(\xi)=\psi_{k, \tau}(\xi) \hat{f}(\xi), \xi \in \mathbb{R}^{n}$. Then, noting $\sum_{j \in \mathbb{Z}} \psi_{j}(\xi)=1(\xi \neq 0)$ and $\sum_{\ell=-1}^{1} \psi_{j+\ell}(\xi)=1$ on supp $\psi_{j}$, we have

$$
T_{\Omega, h, \phi} f(x)=\sum_{k \in \mathbb{Z}} \sum_{j \in \mathbb{Z}} \sum_{\ell=-1}^{1} S_{j-k+\ell, \tau}\left(\sigma_{\Omega, h, \phi, k} * S_{j-k, \tau} f\right)(x)=\sum_{j \in \mathbb{Z}} Q_{j} f(x),
$$

where

$$
Q_{j} f(x)=\sum_{k \in \mathbb{Z}} \sum_{\ell=-1}^{1} S_{j-k+\ell, \tau}\left(\sigma_{\Omega, h, \phi, k} * S_{j-k, \tau} f\right)(x) .
$$


We follow the proof of Theorem 1 in [8], using our Lemma 2.7 and Lemma 2.4 in place of Lemma 2.2 and Lemma 2.4 in [8], respectively, and we see that if $\alpha \in \mathbb{R}$ and $\left(\frac{1}{p}, \frac{1}{q}\right)$ belongs to the interior of the octagon $P_{1} P_{2} R_{2} P_{3} P_{4} P_{5} R_{4} P_{6}$, then we have

$$
\left\|Q_{j} f\right\|_{\dot{F}_{p, q}^{\alpha}\left(\mathbb{R}^{n}\right)} \leq C\|f\|_{\dot{F}_{p, q}^{\alpha}\left(\mathbb{R}^{n}\right)}
$$

About $L^{2}$ estimate, we have

$$
\left\|Q_{j} f\right\|_{\dot{F}_{2,2}^{0}\left(\mathbb{R}^{n}\right)} \leq C a^{-|j| / \max \left(\gamma^{\prime}, 2\right)}\|f\|_{\dot{F}_{2,2}^{0}\left(\mathbb{R}^{n}\right)} .
$$

In fact, by Lemma 2.5 , we get

$$
\begin{aligned}
& \left|\hat{\sigma}_{\Omega, h, \phi, k}(\xi)\right| \leq C\|h\|_{\Delta_{1}}\|\Omega\|_{L^{1}\left(S^{n-1}\right)}, \\
& \left|\hat{\sigma}_{\Omega, h, \phi, k}(\xi, \eta)\right| \leq C\|h\|_{\Delta_{1}} \phi\left(2^{k}\right)\left|A_{\tau}(\xi)\right|
\end{aligned}
$$

and

$$
\left|\hat{\sigma}_{\Omega, h, \phi, \phi, k}(\xi)\right| \leq \frac{C\|h\|_{\Delta_{\gamma}}}{\left(\phi\left(2^{k-1}\right)\left|A_{\tau}(\xi)\right|\right)^{1 / \max \left(\gamma^{\prime}, 2\right)}}
$$

Also, we have

$$
\begin{aligned}
\left\|Q_{j} f\right\|_{\dot{F}_{2,2}^{0}\left(\mathbb{R}^{n}\right)} & =\left(\int_{\mathbb{R}^{n}}\left|\sum_{k \in \mathbb{Z}} \sum_{\ell=-1}^{1} S_{j-k+\ell, \tau}\left(\sigma_{\Omega, h, \phi, k} * S_{j-k, \tau} f\right)(x)\right|^{2} d x\right)^{1 / 2} \\
& =\left(\int_{\mathbb{R}^{n}}\left|\sum_{k \in \mathbb{Z}} \sum_{\ell=-1}^{1} \psi_{j-k+\ell}\left(A_{\tau}(\xi)\right) \hat{\sigma}_{\Omega, h, \phi, k}(\xi) \psi_{j-k}\left(A_{\tau}(\xi)\right) \hat{f}(\xi)\right|^{2} d \xi\right)^{1 / 2} .
\end{aligned}
$$

So, for $j \geq 0$, we have, using (2.33) and $\phi\left(2^{\ell}\right)=1 / a_{-\ell}$ and $a_{\ell+1} / a_{\ell} \geq a=2^{1 /\|\varphi\|_{L} \infty\left(\mathbb{R}_{+}\right)}$,

$$
\begin{aligned}
& \left\|Q_{j} f\right\|_{\dot{F}_{2,2}^{0}\left(\mathbb{R}^{n}\right)} \\
& \quad \leq C\left(\sum_{k \in \mathbb{Z}} \int_{a_{j-k} \leq\left|A_{\tau}(\xi)\right| \leq a_{j-k+2}}\left|\hat{\sigma}_{\Omega, h, \phi, k}(\xi) \hat{f}(\xi)\right|^{2} d \xi\right)^{1 / 2} \\
& \quad \leq C\|h\|_{\Delta_{\gamma}}\left(\sum_{k \in \mathbb{Z}} \int_{a_{j-k} \leq\left|A_{\tau}(\xi)\right| \leq a_{j-k+2}}\left(\frac{\left|A_{\tau}(\xi)\right|}{a_{-k+1}}\right)^{-2 / \max \left(\gamma^{\prime}, 2\right)}|\hat{f}(\xi)|^{2} d \xi\right)^{1 / 2} \\
& \quad \leq C\|h\|_{\Delta_{\gamma}} a^{-(j-1) / \max \left(\gamma^{\prime}, 2\right)}\left(\sum_{k \in \mathbb{Z}} \int_{a_{j-k} \leq\left|A_{\tau}(\xi)\right| \leq a_{j-k+2}}\left(\frac{\left|A_{\tau}(\xi)\right|}{a_{j-k}}\right)^{-2 / \max \left(\gamma^{\prime}, 2\right)}|\hat{f}(\xi)|^{2} d \xi\right)^{1 / 2} \\
& \quad \leq C\|h\|_{\Delta_{\gamma}} a^{-j / \max \left(\gamma^{\prime}, 2\right)}\left(\int_{\mathbb{R}^{n}}|\hat{f}(\xi)|^{2} d \xi\right)^{1 / 2} \\
& \leq C a^{-j / \max \left(\gamma^{\prime}, 2\right)}\|f\|_{\dot{F}_{2,2}^{0}\left(\mathbb{R}^{n}\right)} .
\end{aligned}
$$

In the fourth inequality we used $a_{j+1} \leq c_{1} a_{j}$. 
For $j \leq-1$, using (2.32) we get as before

$$
\begin{aligned}
\left\|Q_{j} f\right\|_{\dot{F}_{2,2}^{0}\left(\mathbb{R}^{n}\right)} & \leq C\left(\sum_{k \in \mathbb{Z}} \int_{a_{j-k} \leq\left|A_{\tau}(\xi)\right| \leq a_{j-k+2}}\left|\hat{\sigma}_{\Omega, \psi, h, k}(\xi) \hat{f}(\xi)\right|^{2} d \xi\right)^{1 / 2} \\
& \leq C\|h\|_{\Delta_{\gamma}}\left(\sum_{k \in \mathbb{Z}} \int_{a_{j-k} \leq\left|A_{\tau}(\xi)\right| \leq a_{j-k+2}}\left(\frac{\left|A_{\tau}(\xi)\right|}{a_{-k}}\right)^{2}|\hat{f}(\xi)|^{2} d \xi\right)^{1 / 2} \\
& \leq C a^{j}\left(\sum_{k \in \mathbb{Z}} \int_{a_{j-k} \leq\left|A_{\tau}(\xi)\right| \leq a_{j-k+2}}\left(\frac{\left|A_{\tau}(\xi)\right|}{a_{j-k}}\right)^{2}|\hat{f}(\xi)|^{2} d \xi\right)^{1 / 2} \\
& \leq C a^{j}\left(\sum_{k \in \mathbb{Z}} \int_{a_{j-k} \leq\left|A_{\tau}(\xi)\right| \leq a_{j-k+2}}|\hat{f}(\xi)|^{2} d \xi\right)^{1 / 2} \\
& \leq C a^{j}\left(\int_{\mathbb{R}^{n}}|\hat{f}(\xi)|^{2} d \xi\right)^{1 / 2} \\
& \leq C a^{j}\|f\|_{L^{2}\left(\mathbb{R}, \dot{F}_{2,2}^{0}\left(\mathbb{R}^{n}\right)\right)^{.}}
\end{aligned}
$$

Thus we have

$$
\left\|Q_{j} f\right\|_{\dot{F}_{2,2}^{0}\left(\mathbb{R}^{n}\right)} \leq C a^{-|j| / \max \left(\gamma^{\prime}, 2\right)}\|f\|_{\dot{F}_{2,2}^{0}\left(\mathbb{R}^{n}\right)},
$$

which shows the required estimate (2.30).

Interpolating these two cases (2.29) and (2.30), we see that if $\alpha \in \mathbb{R}$ and $\left(\frac{1}{p}, \frac{1}{q}\right)$ belongs to the interior of the octagon $P_{1} P_{2} Q_{2} P_{3} P_{4} P_{5} Q_{4} P_{6}$, then $T_{\Omega, h, \phi}$ is bounded on $\dot{F}_{p, q}^{\alpha}\left(\mathbb{R}^{n}\right)$. This completes the proof of Theorem 1.1(i).

Next, we prove (ii). Let $\left|\frac{1}{2}-\frac{1}{p}\right|<\min \left\{\frac{1}{2}, \frac{1}{\gamma^{\prime}}\right\}, 1<q<\infty$, and $\alpha \in \mathbb{R}$. Then, by Theorem 1.1(ii), $T_{\Omega, h, \phi}$ is bounded on $\dot{F}_{p, p}^{\alpha-1}\left(\mathbb{R}^{n}\right)$ and $\dot{F}_{p, p}^{\alpha+1}\left(\mathbb{R}^{n}\right)$. Since $\left(\dot{F}_{p, p}^{\alpha-1}\left(\mathbb{R}^{n}\right), \dot{F}_{p, p}^{\alpha+1}\left(\mathbb{R}^{n}\right)\right)_{\frac{1}{2}, q}=$ $\dot{B}_{p, q}^{\alpha}\left(\mathbb{R}^{n}\right)$, we see by interpolation that $T_{\Omega, h, \phi}$ is bounded on $\dot{B}_{p, q}^{\alpha}\left(\mathbb{R}^{n}\right)$. This shows (ii) and completes the proof of Theorem 1.1.

\section{Proof of Theorem 1.3}

Let $\sigma_{\Omega, h, \phi, k}, a_{k}, \psi_{k}$, and $S_{k}$ be the same as in the proof of Theorem 1.1. Then, noting $\sum_{j \in \mathbb{Z}} \psi_{j}(\xi)=1(\xi \neq 0)$ and $\sum_{\ell=-1}^{1} \psi_{j+\ell}(\xi)=1$ on $\operatorname{supp} \psi_{j}$, we have

$$
T_{\Omega, h, \phi} f(x)=\sum_{k \in \mathbb{Z}} \sum_{j \in \mathbb{Z}} \sum_{\ell=-1}^{1} S_{j-k+\ell}\left(\sigma_{\Omega, h, \phi, k} * S_{j-k} f\right)(x)=\sum_{j \in \mathbb{Z}} \tilde{Q}_{j} f(x),
$$

where

$$
\tilde{Q}_{j} f(x)=\sum_{k \in \mathbb{Z}} \sum_{\ell=-1}^{1} S_{j-k+\ell}\left(\sigma_{\Omega, h, \phi, k} * S_{j-k} f\right)(x) .
$$

Using our Lemma 2.6 and Lemma 2.4(i) in place of Lemma 2.2 and Lemma 2.4 in [8], respectively, we see, as in the proof of Theorem 1.1, that if $\alpha \in \mathbb{R}$ and $\left(\frac{1}{p}, \frac{1}{q}\right)$ belongs to the interior of the octagon $P_{1} P_{2} R_{2} P_{3} P_{4} P_{5} R_{4} P_{6}$, then we have

$$
\left\|\tilde{Q}_{j} f\right\|_{\dot{F}_{p, q}^{\alpha}\left(\mathbb{R}^{n}\right)} \leq C\|f\|_{\dot{F}_{p, q}^{\alpha}\left(\mathbb{R}^{n}\right)} .
$$


Next, we approach the above estimate (3.3) by another method. We calculate the $\dot{F}_{p, q}^{\alpha}$ norm of $\tilde{Q}_{j}$ more directly. Considering the support property of $\psi_{k}$, we have

$$
\begin{aligned}
\left\|\tilde{Q}_{j} f\right\|_{\dot{F}_{p, q}^{\alpha}\left(\mathbb{R}^{n)}\right)} & \left\|\left(\sum_{m \in \mathbb{Z}} a_{m}^{\alpha q}\left|S_{m} \sum_{k \in \mathbb{Z}} \sum_{\ell=-1}^{1} S_{j-k+\ell}\left(\sigma_{\Omega, h, \phi, k} * S_{j-k} f\right)\right|^{q}\right)^{\frac{1}{q}}\right\|_{L^{p}\left(\mathbb{R}^{n}\right)} \\
\leq & \left\|\left(\sum_{m \in \mathbb{Z}} a_{m}^{\alpha q}\left|S_{m} \sum_{\ell=-1}^{1} S_{m+\ell}\left(\sigma_{\Omega, h, \phi, j-m} * S_{m} f\right)\right|^{q}\right)^{\frac{1}{q}}\right\|_{L^{p}\left(\mathbb{R}^{n}\right)} \\
& \times\left\|\left(\sum_{m \in \mathbb{Z}} a_{m}^{\alpha q}\left|S_{m} \sum_{\ell=0}^{1} S_{m+\ell}\left(\sigma_{\Omega, h, \phi, j-m-1} * S_{m+1} f\right)\right|^{q}\right)^{\frac{1}{q}}\right\|_{L^{p}\left(\mathbb{R}^{n}\right)} \\
& \times\left\|\left(\sum_{m \in \mathbb{Z}} a_{m}^{\alpha q}\left|S_{m} \sum_{\ell=-1}^{0} S_{m+\ell}\left(\sigma_{\Omega, h, \phi, j-m-1} * S_{m-1} f\right)\right|^{q}\right)^{\frac{1}{q}}\right\|_{L^{p}\left(\mathbb{R}^{n}\right)} .
\end{aligned}
$$

By Fefferman-Stein's vector-valued inequality for maximal functions, Lemma 2.4(ii), and $a_{m+1} / c_{1} \leq a_{m} \leq a_{m+1} / a$, we get

$$
\begin{aligned}
\left\|\tilde{Q}_{j} f\right\|_{\dot{F}_{p, q}^{\alpha}\left(\mathbb{R}^{n}\right)} & \leq C \sum_{\ell=-1}^{1}\left\|\left(\sum_{m \in \mathbb{Z}} a_{m}^{\alpha q}\left|\sigma_{\Omega, h, \phi, j-m} * S_{m+\ell} f\right|^{q}\right)^{\frac{1}{q}}\right\|_{L^{p}\left(\mathbb{R}^{n}\right)} \\
& \leq C \sum_{\ell=-1}^{1}\left\|\left(\sum_{m \in \mathbb{Z}} a_{m}^{\alpha q}\left|S_{m+\ell} f\right|^{q}\right)^{\frac{1}{q}}\right\|_{L^{p}\left(\mathbb{R}^{n}\right)} \\
& \leq C\left\|\left(\sum_{m \in \mathbb{Z}} a_{m}^{\alpha q}\left|S_{m} f\right|^{q}\right)^{\frac{1}{q}}\right\|_{L^{p}\left(\mathbb{R}^{n)}\right.} \leq C\|f\|_{\dot{F}_{p, q}^{\alpha}\left(\mathbb{R}^{n}\right)}
\end{aligned}
$$

if $\alpha \in \mathbb{R}$ and $\left(\frac{1}{p}, \frac{1}{q}\right)$ belongs to the interior of the parallelogram $Q_{1} Q_{2} Q_{3} Q_{4}$.

Interpolating (3.3) and (3.4), we obtain

$$
\left\|\tilde{Q}_{j} f\right\|_{\dot{F}_{p, q}^{\alpha}\left(\mathbb{R}^{n}\right)} \leq C\|f\|_{\dot{F}_{p, q}^{\alpha}\left(\mathbb{R}^{n}\right)}
$$

if $\alpha \in \mathbb{R}$ and $\left(\frac{1}{p}, \frac{1}{q}\right)$ belongs to the interior of the octagon $Q_{1} Q_{2} R_{2} P_{3} Q_{3} Q_{4} R_{4} P_{6}$ (hexagon $Q_{1} Q_{2} P_{3} Q_{3} Q_{4} P_{6}$ in the case $1<\gamma \leq 2$ ).

About $L^{2}$ estimate, we have

$$
\left\|\tilde{Q}_{j} f\right\|_{\dot{F}_{2,2}^{0}\left(\mathbb{R}^{n}\right)} \leq C\left(\frac{1}{1+|j|}\right)^{\beta / \max \left(\gamma^{\prime}, 2\right)} .
$$

In fact, let $\sigma_{k}=\sigma_{\Omega, h, \phi, k}$. Then we have

$$
\hat{\sigma}_{k}(\xi)=\int_{2^{k-1}}^{2^{k}} \int_{S^{n-1}} \Omega\left(y^{\prime}\right) h(r) e^{-i \phi(r) y^{\prime} \cdot \xi} d \sigma\left(y^{\prime}\right) \frac{d r}{r} .
$$

First we have

$$
\left|\hat{\sigma}_{k}(\xi)\right| \leq 2\|h\|_{\Delta_{\gamma}}\|\Omega\|_{L^{1}\left(S^{n-1}\right)} .
$$


Next, using Hölder's inequality and assuming $\|\Omega\|_{L^{1}\left(S^{n-1}\right)} \leq 1$ without loss of generality, we have

$$
\begin{aligned}
\left|\hat{\sigma}_{k}(\xi)\right| \leq & \left(\int_{2^{k-1}}^{2^{k}}|h(r)|^{\gamma} \frac{d r}{r}\right)^{1 / \gamma}\left(\int_{2^{k-1}}^{2^{k}}\left|\int_{S^{n-1}} \Omega\left(y^{\prime}\right) e^{-i \phi(r) y^{\prime} \cdot \xi} d \sigma\left(y^{\prime}\right)\right|^{\gamma^{\prime}} \frac{d r}{r}\right)^{1 / \gamma^{\prime}} \\
\leq & 2\|h\|_{\Delta_{\gamma}}\left(\int_{2^{k-1}}^{2^{k}}\left|\int_{S^{n-1}} \Omega\left(y^{\prime}\right) e^{-i \phi(r) y^{\prime} \cdot \xi} d \sigma\left(y^{\prime}\right)\right|^{2} \frac{d r}{r}\right)^{\frac{1}{\max \left(2, \gamma^{\prime}\right)}} \\
= & 2\|h\|_{\Delta_{\gamma}}\left(\int_{\phi\left(2^{k-1}\right)}^{\phi\left(2^{k}\right)}\left|\int_{S^{n-1}} \Omega\left(y^{\prime}\right) e^{-i r y^{\prime} \cdot \xi} d \sigma\left(y^{\prime}\right)\right|^{2} \frac{\phi\left(\phi^{-1}(r)\right)}{\phi^{-1}(r) \phi^{\prime}\left(\phi^{-1}(r)\right)} \frac{d r}{r}\right)^{\frac{1}{\max \left(2, \gamma^{\prime}\right)}} \\
\leq & 2\|h\|_{\Delta_{\gamma}}\|\varphi\|_{L^{\infty}\left(\mathbb{R}_{+}\right)}\left(\int_{\phi\left(2^{k-1}\right)}^{\phi\left(2^{k}\right)}\left|\int_{S^{n-1}} \Omega\left(y^{\prime}\right) e^{-i r y^{\prime} \cdot \xi} d \sigma\left(y^{\prime}\right)\right|^{2} \frac{d r}{r}\right)^{\frac{1}{\max \left(2, \gamma^{\prime}\right)}} \\
= & 2\|h\|_{\Delta_{\gamma}}\|\varphi\|_{L^{\infty}\left(\mathbb{R}_{+}\right)} \\
& \times\left(\int_{S^{n-1}} \int_{S^{n-1}} \Omega\left(y^{\prime}\right) \overline{\Omega\left(z^{\prime}\right)} \int_{\phi\left(2^{k-1}\right)}^{\phi\left(2^{k}\right)} e^{-i r\left(y^{\prime}-z^{\prime}\right) \cdot \xi} \frac{d r}{r} d \sigma\left(y^{\prime}\right) d \sigma\left(z^{\prime}\right)\right)^{\frac{1}{\max \left(2, \gamma^{\prime}\right)}} .
\end{aligned}
$$

We see that

$$
\left|\int_{\phi\left(2^{k-1}\right)}^{\phi\left(2^{k}\right)} e^{-i r\left(y^{\prime}-z^{\prime}\right) \cdot \xi} \frac{d r}{r}\right| \leq \log \frac{\phi\left(2^{k}\right)}{\phi\left(2^{k-1}\right)} \leq \log c_{1} .
$$

We see also

$$
\left|\int_{\phi\left(2^{k-1}\right)}^{\phi\left(2^{k}\right)} e^{-i r\left(y^{\prime}-z^{\prime}\right) \cdot \xi} \frac{d r}{r}\right| \leq \frac{2}{\phi\left(2^{k-1}\right)|\xi|\left|\xi^{\prime} \cdot\left(x^{\prime}-y^{\prime}\right)\right|} .
$$

So, as in [10], p.458 (using Lemma 3.1 in [12]), we have for $\beta>1$,

$$
\begin{aligned}
& \left|\int_{\phi\left(2^{k-1}\right)}^{\phi\left(2^{k}\right)} e^{-i r\left(y^{\prime}-z^{\prime}\right) \cdot \xi} \frac{d r}{r}\right| \\
& \quad \leq \frac{C}{\log ^{\beta}\left(\log c_{1}\right) e \phi\left(2^{k-1}\right)|\xi|} \log ^{\beta} \frac{2 e}{\left|\left(y^{\prime}-z^{\prime}\right) \cdot \xi^{\prime}\right|} \quad \text { for } \phi\left(2^{k}\right)|\xi| \geq \frac{c_{1}}{\log c_{1}} .
\end{aligned}
$$

Hence we have

$$
\left|\hat{\sigma}_{k}(\xi)\right| \leq \frac{2\|h\|_{\Delta_{\gamma}}\left(W \mathcal{F}_{\beta}(\Omega)\right)^{2 / \max \left\{\gamma^{\prime}, 2\right\}}\|\Omega\|_{L^{1}\left(S^{n-1}\right)}^{1-2 / \max \left\{\gamma^{\prime}, 2\right\}}}{\left(\log \left(e\left(\log c_{1}\right) \phi\left(2^{k}\right)|\xi| / c_{1}\right)\right)^{\beta / \max \left\{\gamma^{\prime}, 2\right\}}}
$$

for $\phi\left(2^{k}\right)|\xi| \geq \frac{c_{1}}{\log c_{1}} \geq e$.

On the other hand, using the cancelation property of $\Omega$, we get easily

$$
\left|\hat{\sigma}_{k}(\xi)\right| \leq 2\|h\|_{\Delta_{1}}\|\Omega\|_{L^{1}\left(S^{n-1}\right)} \phi\left(2^{k}\right)|\xi| .
$$

Now we can estimate the $L^{2}$ norm of $\tilde{Q}_{j} f$ :

$$
\begin{aligned}
\left\|\tilde{Q}_{j} f\right\|_{\dot{F}_{2,2}^{0}\left(\mathbb{R}^{n}\right)} & =\left(\int_{\mathbb{R}^{n}}\left|\sum_{k \in \mathbb{Z}} \sum_{\ell=-1}^{1} S_{j-k+\ell}\left(\sigma_{\Omega, h, \phi, k} * S_{j-k} f\right)(x)\right|^{2} d x\right)^{1 / 2} \\
& =\left(\int_{\mathbb{R}^{n}}\left|\sum_{k \in \mathbb{Z}} \sum_{\ell=-1}^{1} \psi_{j-k+\ell}(\xi) \hat{\sigma}_{\Omega, h, \phi, k}(\xi) \psi_{j-k}(\xi) \hat{f}(\xi)\right|^{2} d \xi\right)^{1 / 2} .
\end{aligned}
$$


Note that $\phi\left(2^{k}\right)|\xi|=|\xi| / a_{-k} \geq a^{j}|\xi| / a_{j-k} \geq a^{j}$ for $a_{j-k} \leq|\xi| \leq a_{j-k+2}$ and $j \geq 0$, where $a_{\ell+1} / a_{\ell} \geq a=2^{1 /\|\varphi\|_{L}{ }_{(\mathbb{R}+)}}$. So, for $j \geq 2 \log _{a}\left(c_{1} / \log c_{1}\right)$ and $a_{j-k} \leq|\xi| \leq a_{j-k+2}$, we have

$$
\frac{\log c_{1}}{c_{1}} \phi\left(2^{k}\right)|\xi| \geq a^{\frac{j}{2}} \geq 1
$$

and hence we have

$$
\begin{aligned}
\left\|\tilde{Q}_{j} f\right\|_{\dot{F}_{2,2}^{0}\left(\mathbb{R}^{n}\right)} & \leq C\left(\sum_{k \in \mathbb{Z}} \int_{a_{j-k} \leq|\xi| \leq a_{j-k+2}}\left|\hat{\sigma}_{\Omega, h, \phi, k}(\xi) \hat{f}(\xi)\right|^{2} d \xi\right)^{1 / 2} \\
& \leq C\|h\|_{\Delta_{\gamma}}\left(\sum_{k \in \mathbb{Z}} \int_{a_{j-k} \leq|\xi| \leq a_{j-k+2}}\left(\frac{1}{1+j}\right)^{2 \beta / \max \left(\gamma^{\prime}, 2\right)}|\hat{f}(\xi)|^{2} d \xi\right)^{1 / 2} \\
& \leq C\|h\|_{\Delta_{\gamma}}\left(\frac{1}{1+j}\right)^{\beta / \max \left(\gamma^{\prime}, 2\right)}\left(\sum_{k \in \mathbb{Z}} \int_{a_{j-k} \leq|\xi| \leq a_{j-k+2}}|\hat{f}(\xi)|^{2} d \xi\right)^{1 / 2} \\
& \leq C\|h\|_{\Delta_{\gamma}}\left(\frac{1}{1+j}\right)^{\beta / \max \left(\gamma^{\prime}, 2\right)}\left(\int_{\mathbb{R}^{n}}|\hat{f}(\xi)|^{2} d \xi\right)^{1 / 2} \\
& \leq C\left(\frac{1}{1+j}\right)^{\beta / \max \left(\gamma^{\prime}, 2\right)}\|f\|_{\dot{F}_{2,2}^{0}\left(\mathbb{R}^{n}\right)} .
\end{aligned}
$$

For $j \leq-2$, we have $\phi\left(2^{k}\right)|\xi|=|\xi| / a_{-k} \leq a^{2+j}$ for $a_{j-k} \leq|\xi| \leq a_{j-k+2}$. So, using (3.9) we get as before

$$
\begin{aligned}
\left\|\tilde{Q}_{j} f\right\|_{\dot{F}_{2,2}^{0}\left(\mathbb{R}^{n}\right)} & \leq C\left(\sum_{k \in \mathbb{Z}} \int_{a_{j-k} \leq|\xi| \leq a_{j-k+2}}\left|\hat{\sigma}_{\Omega, \psi, h, k}(\xi) \hat{f}(\xi)\right|^{2} d \xi\right)^{1 / 2} \\
& \leq C\|h\|_{\Delta_{\gamma}}\left(\sum_{k \in \mathbb{Z}} \int_{a_{j-k} \leq|\xi| \leq a_{j-k+2}} a^{4+2 j}|\hat{f}(\xi)|^{2} d \xi\right)^{1 / 2} \\
& \leq C a^{j}\left(\sum_{k \in \mathbb{Z}} \int_{a_{j-k} \leq|\xi| \leq a_{j-k+2}}|\hat{f}(\xi)|^{2} d \xi\right)^{1 / 2} \\
& \leq C a^{j}\left(\int_{\mathbb{R}^{n}}|\hat{f}(\xi)|^{2} d \xi\right)^{1 / 2} \\
& \leq C a^{j}\|f\|_{\dot{F}_{2,2}^{0}\left(\mathbb{R}^{n}\right)} .
\end{aligned}
$$

For $-2 \leq j<2 \log _{a}\left(c_{1} / \log c_{1}\right)$, using (3.7), we get

$$
\left\|\tilde{Q}_{f} f\right\|_{\dot{F}_{2,2}^{0}\left(\mathbb{R}^{n}\right)} \leq C\left(\sum_{k \in \mathbb{Z}} \int_{a_{j-k} \leq|\xi| \leq a_{j-k+2}}|\hat{f}(\xi)|^{2} d \xi\right)^{1 / 2} \leq C\|f\|_{\dot{F}_{2,2}^{0}\left(\mathbb{R}^{n}\right)}
$$

Thus we have (3.6) for $j \in \mathbb{Z}$. Now, let $\mathcal{Q}_{1}=\left(\frac{\max \left(\gamma^{\prime}, 2\right)}{2 \beta}, \frac{\max \left(\gamma^{\prime}, 2\right)}{2 \beta}\right), \mathcal{Q}_{2}=\left(\frac{1}{\gamma^{\prime}}+\frac{\max \left(\gamma^{\prime}, 2\right)}{\beta}\left(\frac{1}{2}-\right.\right.$ $\left.\left.\frac{1}{\gamma^{\prime}}\right), \frac{\max \left(\gamma^{\prime}, 2\right)}{2 \beta}\right), \mathcal{P}_{3}=\left(\frac{1}{2}+\frac{1}{\max \left(\gamma^{\prime}, 2\right)}-\frac{1}{\beta}, \frac{1}{2}\right), \mathcal{Q}_{3}=\left(1-\frac{\max \left(\gamma^{\prime}, 2\right)}{2 \beta}, 1-\frac{\max \left(\gamma^{\prime}, 2\right)}{2 \beta}\right), \mathcal{Q}_{4}=\left(\frac{1}{\gamma}-\frac{\max \left(\gamma^{\prime}, 2\right)}{\beta}\left(\frac{1}{\gamma}-\right.\right.$ $\left.\left.\frac{1}{2}\right), 1-\frac{\max \left(\gamma^{\prime}, 2\right)}{2 \beta}\right), \mathcal{P}_{6}=\left(\frac{1}{2}-\frac{1}{\max \left(\gamma^{\prime}, 2\right)}+\frac{1}{\beta}, \frac{1}{2}\right), \mathcal{R}_{2}=\left(1-\frac{1}{2 \gamma}-\frac{\max \left(\gamma^{\prime}, 2\right)}{2 \beta \gamma^{\prime}}, \frac{1}{2 \gamma}+\frac{\max \left(\gamma^{\prime}, 2\right)}{2 \beta \gamma^{\prime}}\right)$, and $\mathcal{R}_{4}=\left(\frac{1}{2 \gamma}+\frac{\max \left(\gamma^{\prime}, 2\right)}{2 \beta \gamma^{\prime}}, 1-\frac{1}{2 \gamma}-\frac{\max \left(\gamma^{\prime}, 2\right)}{2 \beta \gamma^{\prime}}\right)$. Then, for $\left(\frac{1}{p}, \frac{1}{q}\right)$ belonging to the interior of the octagon $\mathcal{Q}_{1} \mathcal{Q}_{2} \mathcal{R}_{2} \mathcal{P}_{3} \mathcal{Q}_{3} \mathcal{Q}_{4} \mathcal{R}_{4} \mathcal{P}_{6}$ (hexagon $\mathcal{Q}_{1} \mathcal{Q}_{2} \mathcal{P}_{3} \mathcal{Q}_{3} \mathcal{Q}_{4} \mathcal{P}_{6}$ in the case $1<\gamma \leq 2$ ), we can find $\left(\frac{1}{p_{1}}, \frac{1}{q_{1}}\right)$ in the interior of the octagon $Q_{1} Q_{2} R_{2} P_{3} Q_{3} Q_{4} R_{4} P_{6}$ (hexagon $Q_{1} Q_{2} P_{3} Q_{3} Q_{4} P_{6}$ 
in the case $1<\gamma \leq 2)$ such that $\frac{1}{p}=\frac{\theta}{2}+\frac{1-\theta}{p_{1}}, \frac{1}{q}=\frac{\theta}{2}+\frac{1-\theta}{q_{1}}$, and $1>\theta>\frac{\max \left(\gamma^{\prime}, 2\right)}{\beta}$. Hence, for $\alpha \in \mathbb{R}$, taking $\alpha_{1}$ with $\alpha=(1-\theta) \alpha_{1}$ and interpolating between (3.6) and (3.5), we obtain the desired estimate

$$
\left\|\tilde{Q}_{j} f\right\|_{\dot{F}_{p, q}^{\alpha}\left(\mathbb{R}^{n}\right)} \leq C(1+|j|)^{-\theta \beta / \max \left\{\gamma^{\prime}, 2\right\}}\|f\|_{\dot{F}_{p, q}^{\alpha}\left(\mathbb{R}^{n}\right)}
$$

Summing up this with respect to $j$, we finish the proof of Theorem 1.3(i). The proof of (ii) is the same as in Theorem 1.1(i).

\section{Proof of Theorem 1.2}

In this section we shall give the proof of Theorem 1.2.

(A) $L \log L$ case. Let $\Omega \in L \log L\left(S^{n-1}\right)$ satisfying the cancelation property. Then letting $A_{m}=\left\|\Omega \chi_{2^{m-1} \leq\left|\Omega\left(y^{\prime}\right)\right|<2^{m}}\right\|_{L^{1}\left(S^{n-1}\right)}$ and $\Lambda=\left\{m \in \mathbb{N}: A_{m}>2^{-m}\right\}$, we can construct $\Omega_{m} \in$ $L^{2}\left(S^{n-1}\right)(m \in \Lambda)$ and $\Omega_{0} \in \bigcap_{1<r<2} L^{r}\left(S^{n-1}\right)$ such that

$$
\begin{aligned}
& \left\|\Omega_{m}\right\|_{L^{2}\left(S^{n-1}\right)} \leq C 2^{m}, \quad\left\|\Omega_{m}\right\|_{L^{1}\left(S^{n-1}\right)} \leq C, \\
& \sum_{m \in \Lambda} m A_{m} \leq C\|\Omega\|_{L \log L\left(S^{n-1}\right)}, \\
& \int_{S^{n-1}} \Omega_{m}\left(y^{\prime}\right) d \sigma\left(y^{\prime}\right)=0 \quad(m=0, m \in \Lambda), \quad \Omega=\Omega_{0}+\sum_{m \in \Lambda} A_{m} \Omega_{m} .
\end{aligned}
$$

From the above, we see that

$$
T_{\Omega, \psi, h} f=T_{\Omega_{0}, h, \phi} f+\sum_{m \in \Lambda} A_{m} T_{\Omega_{m}, h, \phi} f
$$

So, we consider $T_{\Omega_{m}, h, \phi}$. We use the notations in Section 3 with minor change such as $\tilde{Q}_{m, j}$ for $\Omega_{m}$ instead of $\tilde{Q}_{j}$ for $\Omega$. Since $\left\|\Omega_{m}\right\|_{L^{1}\left(S^{n-1}\right)} \leq C$, we have as in Section 3 that

$$
\left\|\tilde{Q}_{m, j} f\right\|_{\dot{F}_{p, q}^{\alpha}\left(\mathbb{R}^{n}\right)} \leq C\|f\|_{\dot{F}_{p, q}^{\alpha}\left(\mathbb{R}^{n}\right)}
$$

if $\alpha \in \mathbb{R}$ and $\left(\frac{1}{p}, \frac{1}{q}\right)$ belongs to the interior of the octagon $Q_{1} Q_{2} R_{2} P_{3} Q_{3} Q_{4} R_{4} P_{6}$ (hexagon $Q_{1} Q_{2} P_{3} Q_{3} Q_{4} P_{6}$ in the case $1<\gamma \leq 2$ ).

About $L^{2}$ estimate, we have

$$
\left\|\tilde{Q}_{m, j} f\right\|_{\dot{F}_{2,2}^{0}\left(\mathbb{R}^{n}\right)} \leq C a^{-\frac{\beta}{m}|j|}\|f\|_{\dot{F}_{2,2}^{0}\left(\mathbb{R}^{n}\right)}
$$

for some $\beta$ with $0<\beta<1 / 2$. In fact, let $\sigma_{m, k}=\sigma_{\Omega_{m}, h, \phi, k}$. Since $\left\|\Omega_{m}\right\|_{L^{1}\left(S^{n-1}\right)} \leq C$ and $\left\|\Omega_{m}\right\|_{L^{2}\left(S^{n-1}\right)} \leq C 2^{m}$, we get by Lemma 3.1 in [23], p.1567,

$$
\begin{aligned}
\left|\hat{\sigma}_{m, k}(\xi)\right| & \leq C\|h\|_{\Delta_{1}}, \\
\left|\hat{\sigma}_{m, k}(\xi)\right| & \leq C \frac{\|h\|_{\Delta_{\gamma}}\left(1+\|\varphi\|_{\infty}\right)}{\left|\phi\left(2^{k-1}\right) \xi\right|^{\frac{\beta}{m}}}, \\
\left|\hat{\sigma}_{m, k}(\xi)\right| & \leq C\|h\|_{\Delta_{1}}\left|\phi\left(2^{k}\right) \xi\right|^{\frac{\beta}{m}}
\end{aligned}
$$


where $\beta$ is a fixed constant with $0<\beta<1 / 2$. Using Plancherel's theorem, (4.8), the support property of $\psi_{j}$, and $a_{k+1} / c_{1} \leq a_{k} \leq a_{k+1} / a$, we get for $j \geq 0$,

$$
\begin{aligned}
\left\|\tilde{Q}_{m, j} f\right\|_{\dot{F}_{2,2}^{0}\left(\mathbb{R}^{n}\right)} & \leq C\left(\sum_{k \in \mathbb{Z}} \int_{a_{j-k} \leq|\xi|<a_{j-k+2}}\left|\hat{\sigma}_{m, k}(\xi) \hat{f}(\xi)\right|^{2} d \xi\right)^{\frac{1}{2}} \\
& \leq C\left(\sum_{k \in \mathbb{Z}} \int_{a_{j-k} \leq|\xi|<a_{j-k+2}} \frac{1}{\left|\phi\left(2^{k-1}\right) \xi\right|^{\frac{\beta}{m}}}|\hat{f}(\xi)|^{2} d \xi\right)^{\frac{1}{2}} \\
& \leq C a^{-\frac{\beta}{m} j}\left(\sum_{k \in \mathbb{Z}} \int_{a_{j-k} \leq|\xi|<a_{j-k+2}}|\hat{f}(\xi)|^{2} d \xi\right)^{\frac{1}{2}} \\
& \leq C a^{-\frac{\beta}{m} j}\|f\|_{\dot{F}_{2,2}^{0}\left(\mathbb{R}^{n}\right)} .
\end{aligned}
$$

For $j<0$, using (4.9) in place of (4.8), we get

$$
\left\|\tilde{Q}_{m, j} f\right\|_{\dot{F}_{2,2}^{0}\left(\mathbb{R}^{n}\right)} \leq C a^{\frac{\beta}{m} j}\|f\|_{\dot{F}_{2,2}^{0}\left(\mathbb{R}^{n}\right)} .
$$

This shows (4.6). Interpolating (4.6) and (4.5), we obtain for some $0<\theta<1$,

$$
\left\|\tilde{Q}_{m, j} f\right\|_{\dot{F}_{p, q}^{\alpha}\left(\mathbb{R}^{n}\right)} \leq C a^{-\frac{\beta \theta}{m}|j|}\|f\|_{\dot{F}_{p, q}^{\alpha}\left(\mathbb{R}^{n}\right)}
$$

provided $\alpha \in \mathbb{R}$ and $\left(\frac{1}{p}, \frac{1}{q}\right)$ belongs to the interior of the octagon $Q_{1} Q_{2} R_{2} P_{3} Q_{3} Q_{4} R_{4} P_{6}$ (hexagon $Q_{1} Q_{2} P_{3} Q_{3} Q_{4} P_{6}$ in the case $1<\gamma \leq 2$ ).

From (4.10) and the definition of $\tilde{Q}_{m, j}$ it follows

$$
\left\|T_{\Omega_{m}, h, \phi} f\right\|_{\dot{F}_{p, q}^{\alpha}\left(\mathbb{R}^{n}\right)} \leq C \sum_{j \in \mathbb{Z}} a^{-\frac{\beta \theta}{m}|j|}\|f\|_{\dot{F}_{p, q}^{\alpha}\left(\mathbb{R}^{n}\right)} \leq \frac{C}{1-a^{-\frac{\beta \theta}{m}}}\|f\|_{\dot{F}_{p, q}^{\alpha}\left(\mathbb{R}^{n}\right)} \leq C \frac{m}{\beta \theta}\|f\|_{\dot{F}_{p, q}^{\alpha}\left(\mathbb{R}^{n}\right)} .
$$

We can see that the same estimate holds for $\Omega_{0}$. Thus, by (4.4) we have

$$
\left\|T_{\Omega, h, \phi} f\right\|_{\dot{F}_{p, q}^{\alpha}\left(\mathbb{R}^{n}\right)} \leq C\left(1+\sum_{m \in \Lambda} A_{m} m\right)\|f\|_{\dot{F}_{p, q}^{\alpha}\left(\mathbb{R}^{n}\right)} \leq C\|f\|_{\dot{F}_{p, q}^{\alpha}\left(\mathbb{R}^{n}\right)}
$$

provided $\alpha \in \mathbb{R}$ and $\left(\frac{1}{p}, \frac{1}{q}\right)$ belongs to the interior of the octagon $Q_{1} Q_{2} R_{2} P_{3} Q_{3} Q_{4} R_{4} P_{6}$ (hexagon $Q_{1} Q_{2} P_{3} Q_{3} Q_{4} P_{6}$ in the case $1<\gamma \leq 2$ ). This completes the proof of Theorem 1.2 in the case $\Omega \in L \log L\left(S^{n-1}\right)$.

(B) Block space case. Let $r>1$. Then if $\Omega \in B_{r}^{(0,0)}\left(S^{n-1}\right)$ and satisfies the cancelation condition, it can be written as $\Omega=\sum_{\ell=1}^{\infty} \lambda_{\ell} \breve{\Omega}_{\ell}$, where $\lambda_{\ell} \in \mathbb{C}$ and $\breve{\Omega}_{\ell}$ is an $r$-block supported on a cap $B_{\ell}=B\left(x_{\ell}, \tau_{\ell}\right) \cap S^{n-1}$ on $S^{n-1}$ and

$$
\sum_{\ell=1}^{\infty}\left|\lambda_{\ell}\right|\left\{1+\log \left(\left|B_{\ell}\right|^{-1}\right)\right\}<2\|\Omega\|_{B_{r}^{(0,0)}\left(S^{n-1}\right)}<\infty .
$$

To each block $\breve{\Omega}_{\ell}$, we define

$$
\Omega_{\ell}\left(y^{\prime}\right)=\breve{\Omega}_{\ell}\left(y^{\prime}\right)-\frac{1}{\left|S^{n-1}\right|} \int_{S^{n-1}} \breve{\Omega}_{\ell}\left(x^{\prime}\right) d \sigma\left(x^{\prime}\right) .
$$


Let $\Lambda=\left\{\ell \in \mathbb{N} ;\left|B_{\ell}\right| \leq 1 / 2\right\}$ and set

$$
\Omega_{0}=\Omega-\sum_{\ell \in \Lambda} \lambda_{\ell} \Omega_{\ell} .
$$

Then there exists a positive constant $C$ such that the following hold for all $\ell \in \Lambda$ :

$$
\begin{aligned}
& \int_{S^{n-1}} \Omega_{\ell}\left(x^{\prime}\right) d \sigma\left(x^{\prime}\right)=0, \\
& \left\|\Omega_{\ell}\right\|_{L^{r}\left(S^{n-1}\right)} \leq C\left|B_{\ell}\right|^{-1 / r^{\prime}}, \\
& \left\|\Omega_{\ell}\right\|_{L^{1}\left(S^{n-1}\right)} \leq 2, \\
& \Omega=\Omega_{0}+\sum_{\ell \in \Lambda} \lambda_{\ell} \Omega_{\ell} .
\end{aligned}
$$

Moreover, from (4.11) and the definition of $\Omega_{\ell}$ it follows that

$$
\begin{aligned}
& \left\|\Omega_{0}\right\|_{L^{r}\left(S^{n-1}\right)} \leq C \sum_{\ell \in \mathbb{N} \backslash \Lambda} 2^{-1 / r^{\prime}}\left|\lambda_{\ell}\right| \leq C\|\Omega\|_{B_{r}^{(0,0)}\left(S^{n-1}\right)}, \\
& \int_{S^{n-1}} \Omega_{0}\left(x^{\prime}\right) d \sigma\left(x^{\prime}\right)=0 .
\end{aligned}
$$

By (4.16), we have

$$
T_{\Omega, h, \phi} f(x)=\sum_{\ell \in \Lambda \cup 0} \lambda_{\ell} T_{\Omega_{\ell}, h, \phi} f(x) .
$$

So, we have only to show the boundedness of $T_{\Omega_{\ell}, h, \phi} f$. We use the notations in Section 3 with minor change such as $\tilde{Q}_{\ell, j}$ for $\Omega_{\ell}$ instead of $\tilde{Q}_{j}$ for $\Omega$. Since $\left\|\Omega_{\ell}\right\|_{L^{1}\left(S^{n-1}\right)} \leq C$, we have as in Section 3 that

$$
\left\|\tilde{Q}_{\ell, f} f\right\|_{\dot{F}_{p, q}^{\alpha}\left(\mathbb{R}^{n}\right)} \leq C\|f\|_{\dot{F}_{p, q}^{\alpha}\left(\mathbb{R}^{n}\right)}
$$

if $\alpha \in \mathbb{R}$ and $\left(\frac{1}{p}, \frac{1}{q}\right)$ belongs to the interior of the octagon $Q_{1} Q_{2} R_{2} P_{3} Q_{3} Q_{4} R_{4} P_{6}$ (hexagon $Q_{1} Q_{2} P_{3} Q_{3} Q_{4} P_{6}$ in the case $1<\gamma \leq 2$ ).

About $L^{2}$ estimate, we have

$$
\left\|\tilde{Q}_{\ell, j} f\right\|_{\dot{F}_{2,2}^{0}\left(\mathbb{R}^{n}\right)} \leq C a^{-\frac{\beta}{m_{\ell}}|j|}\|f\|_{\dot{F}_{2,2}^{0}\left(\mathbb{R}^{n}\right)}
$$

for some $\beta$ with $0<\beta<r$. In fact, let $\sigma_{\ell, k}=\sigma_{\Omega_{\ell}, h, \phi, k}$. For $\ell \in \Lambda \cup\{0\}$, we set $m_{\ell}=$ $\left[\log _{2}\left|B_{\ell}\right|^{-1 / r^{\prime}}\right]+1$, where $[\cdot]$ denotes the greatest integer function.

Since $\left\|\Omega_{\ell}\right\|_{L^{1}\left(S^{n-1}\right)} \leq 2$ and $\left\|\Omega_{\ell}\right\|_{L^{2}\left(S^{n-1}\right)} \leq C 2^{m_{\ell}}$, we get by Lemma 3.1 in [23], p.1567

$$
\begin{aligned}
&\left|\hat{\sigma}_{\ell, k}(\xi)\right| \leq C\|h\|_{\Delta_{1}}, \\
&\left|\hat{\sigma}_{\ell, k}(\xi)\right| \leq C \frac{\|h\|_{\Delta_{\gamma}}\left(1+\|\varphi\|_{\infty}\right)}{\left|\phi\left(2^{k-1}\right) \xi\right|^{\frac{\beta}{m_{\ell}}}} \\
&\left|\hat{\sigma}_{\ell, k}(\xi)\right| \leq C\|h\|_{\Delta_{1}}\left|\phi\left(2^{k}\right) \xi\right|^{\frac{\beta}{m_{\ell}}},
\end{aligned}
$$


where $\beta$ is a fixed constant with $0<\beta<r$. Using Plancherel's theorem, (4.23), the support property of $\psi_{j}$, and $a_{k+1} / c_{1} \leq a_{k} \leq a_{k+1} / a$, we get for $j \geq 0$,

$$
\begin{aligned}
\left\|\tilde{Q}_{\ell, j} f\right\|_{\dot{F}_{2,2}^{0}\left(\mathbb{R}^{n}\right)} & \leq C\left(\sum_{k \in \mathbb{Z}} \int_{a_{j-k} \leq|\xi|<a_{j-k+2}}\left|\hat{\sigma}_{\ell, k}(\xi) \hat{f}(\xi)\right|^{2} d \xi\right)^{\frac{1}{2}} \\
& \leq C\left(\sum_{k \in \mathbb{Z}} \int_{a_{j-k} \leq|\xi|<a_{j-k+2}} \frac{1}{\left|\phi\left(2^{k-1}\right) \xi\right|^{\frac{\beta}{m_{\ell}}}}|\hat{f}(\xi)|^{2} d \xi\right)^{\frac{1}{2}} \\
& \leq C a^{-\frac{\beta}{m_{\ell}} j}\left(\sum_{k \in \mathbb{Z}} \int_{a_{j-k} \leq|\xi|<a_{j-k+2}}|\hat{f}(\xi)|^{2} d \xi\right)^{\frac{1}{2}} \\
& \leq C a^{-\frac{\beta}{m_{\ell}} j} \mid f \|_{\dot{F}_{2,2}^{0}\left(\mathbb{R}^{n}\right)} .
\end{aligned}
$$

For $j<0$, using (4.9) in place of (4.8), we get

$$
\left\|\tilde{Q}_{\ell, j} f\right\|_{\dot{F}_{2,2}^{0}\left(\mathbb{R}^{n}\right)} \leq C a^{\frac{\beta}{m_{\ell} j}}\|f\|_{\dot{F}_{2,2}^{0}\left(\mathbb{R}^{n}\right)} .
$$

This shows (4.21). Interpolating (4.21) and (4.20), we obtain for some $0<\theta<1$,

$$
\left\|\tilde{Q}_{\ell, f} f\right\|_{\dot{F}_{p, q}^{\alpha}\left(\mathbb{R}^{n}\right)} \leq C a^{-\frac{\beta \theta}{m_{\ell}}|j|}\|f\|_{\dot{F}_{p, q}^{\alpha}\left(\mathbb{R}^{n}\right)}
$$

provided $\alpha \in \mathbb{R}$ and $\left(\frac{1}{p}, \frac{1}{q}\right)$ belongs to the interior of the octagon $Q_{1} Q_{2} R_{2} P_{3} Q_{3} Q_{4} R_{4} P_{6}$ (hexagon $Q_{1} Q_{2} P_{3} Q_{3} Q_{4} P_{6}$ in the case $1<\gamma \leq 2$ ).

From (4.25) and the definition of $\tilde{Q}_{\ell, j}$ it follows

$$
\left\|T_{\Omega_{\ell}, h, \phi} f\right\|_{\dot{F}_{p, q}^{\alpha}\left(\mathbb{R}^{n}\right)} \leq C \sum_{j \in \mathbb{Z}} a^{-\frac{\beta \theta}{m_{\ell}}|j|}\|f\|_{\dot{F}_{p, q}^{\alpha}\left(\mathbb{R}^{n}\right)} \leq \frac{C}{1-a^{-\frac{\beta \theta}{m_{\ell}}}}\|f\|_{\dot{F}_{p, q}^{\alpha}\left(\mathbb{R}^{n}\right)} \leq C \frac{m_{\ell}}{\beta \theta}\|f\|_{\dot{F}_{p, q}^{\alpha}\left(\mathbb{R}^{n}\right)} .
$$

We can see that the same estimate holds for $\Omega_{0}$. Thus, by (4.14) we have

$$
\left\|T_{\Omega, h, \phi} f\right\|_{\dot{F}_{p, q}^{\alpha}\left(\mathbb{R}^{n}\right)} \leq C\left(1+\sum_{\ell \in \Lambda} \lambda_{\ell} m_{\ell}\right)\|f\|_{\dot{F}_{p, q}^{\alpha}\left(\mathbb{R}^{n}\right)} \leq C\|f\|_{\dot{F}_{p, q}^{\alpha}\left(\mathbb{R}^{n}\right)}
$$

provided $\alpha \in \mathbb{R}$ and $\left(\frac{1}{p}, \frac{1}{q}\right)$ belongs to the interior of the octagon $Q_{1} Q_{2} R_{2} P_{3} Q_{3} Q_{4} R_{4} P_{6}$ (hexagon $Q_{1} Q_{2} P_{3} Q_{3} Q_{4} P_{6}$ in the case $1<\gamma \leq 2$ ).

This completes the proof of Theorem 1.2.

\section{Appendix}

In this section we shall prove Lemma 2.6. Let $\left\{a_{j}\right\}_{j \in \mathbb{Z}}, \psi_{j}, \Psi_{j}$, and $S_{j}$ be the same as in Lemma 2.6. Set $\eta_{j}(\xi)=\eta\left(\xi / a_{j+1}\right)$ and $\hat{\Phi}_{j}(\xi)=\eta_{j}(\xi)$. Then we have

$$
(-i x)^{\alpha} \Phi_{j}(x)=c_{n} \int_{\mathbb{R}^{n}} \partial^{\alpha} \eta_{j}(\xi) e^{i x \cdot \xi} d \xi
$$

so we have

$$
\left|x^{\alpha}\right|\left|\Phi_{j}(x)\right| \leq C \int_{\mathbb{R}^{n}}\left|\partial^{\alpha} \eta_{j}(\xi)\right| d \xi \leq C \int_{\text {supp } \partial^{\alpha} \eta_{j}}\left|\partial^{\alpha} \eta_{j}(\xi)\right| d \xi .
$$


From this and the definition of $\eta_{j}$ we get

$$
\left|\Phi_{j}(x)\right| \leq C a^{n} a_{j+1}^{n}
$$

and for $N \in \mathbb{N}$,

$$
|x|^{N}\left|\Phi_{j}(x)\right| \leq C \frac{1}{\left((a-1) a_{j+1}\right)^{N}}\left(\int_{a_{j+1}}^{a a_{j+1}} r^{n-1} d r\right) \leq C \frac{\left(a a_{j+1}\right)^{n}}{\left((a-1) a_{j+1}\right)^{N}} .
$$

Thus, for $N \in \mathbb{N}$ we have

$$
\left|\Phi_{j}(x)\right| \leq C\left(\frac{a}{a-1}\right)^{n} \frac{\left((a-1) a_{j+1}\right)^{n}}{\left(1+\left|(a-1) a_{j+1} x\right|\right)^{N}} .
$$

Next we have

$$
(-i x)^{\alpha} \partial_{x_{k}} \Phi_{j}(x)=c_{n} \int_{\mathbb{R}^{n}} \partial^{\alpha}\left(i \xi_{k} \eta_{j}\right)(\xi) e^{i x \cdot \xi} d \xi
$$

so we have

$$
\left|x^{\alpha}\right|\left|\partial_{x_{k}} \Phi_{j}(x)\right| \leq C \int_{\mathbb{R}^{n}}\left|\partial^{\alpha}\left(\xi_{k} \eta_{j}\right)(\xi)\right| d \xi \leq C \int_{\text {supp } \partial^{\alpha}\left(\xi_{k} \eta_{j}\right)}\left|\partial^{\alpha}\left(\xi_{k} \eta_{j}\right)(\xi)\right| d \xi .
$$

From this and the definition of $\eta_{j}$ we get

$$
\left|\nabla \Phi_{j}(x)\right| \leq C\left(a a_{j+1}\right)^{n+1}
$$

and for $N \in \mathbb{N}$,

$$
\begin{aligned}
|x|^{N}\left|\nabla \Phi_{j}(x)\right| & \leq C \frac{1}{\left((a-1) a_{j+1}\right)^{N-1}} \int_{a_{j+1}}^{a a_{j+1}} r^{n-1} d r+C \frac{1}{\left((a-1) a_{j+1}\right)^{N}} \int_{a_{j+1}}^{a a_{j+1}} r^{n} d r \\
& \leq C \frac{\left(a a_{j+1}\right)^{n}}{\left((a-1) a_{j+1}\right)^{N-1}}+C \frac{\left(a a_{j+1}\right)^{n+1}}{\left((a-1) a_{j+1}\right)^{N}} .
\end{aligned}
$$

Thus, for $N \in \mathbb{N}$ we have

$$
\left|\nabla \Phi_{j}(x)\right| \leq C\left(\frac{a}{a-1}\right)^{n+1} \frac{\left((a-1) a_{j+1}\right)^{n+1}}{\left(1+\left|(a-1) a_{j+1} x\right|\right)^{N}} .
$$

Let $b=a-1$ and $B=\left(\frac{a}{a-1}\right)^{n+1}$. Taking $N=n+1$ and using (A.3) and (A.6), we obtain

$$
\begin{aligned}
I & :=\int_{|x|>2|y|}\left(\sum_{k \in \mathbb{Z}}\left|\Phi_{k}(x-y)-\Phi_{k}(x)\right|^{2}\right)^{1 / 2} d x \\
& \leq \int_{|x|>2|y|} \sum_{k \in \mathbb{Z}}\left|\Phi_{k}(x-y)-\Phi_{k}(x)\right| d x \\
& \leq \sum_{a_{k+1}<|b y|^{-1}} \int_{|x|>2|y|}\left|\Phi_{k}(x-y)-\Phi_{k}(x)\right| d x
\end{aligned}
$$




$$
\begin{aligned}
& +\sum_{a_{k+1} \geq|b y|^{-1}} \int_{|x|>2|y|}\left|\Phi_{k}(x-y)\right|+\left|\Phi_{k}(x)\right| d x \\
\leq & \sum_{a_{k+1}<|b y|^{-1}} \int_{|x|>2|y|}|y|\left|\nabla \Phi_{k}(x-\theta y)\right| d x \\
& +\sum_{a_{k+1} \geq|b y|^{-1}} \int_{|x|>2|y|}\left|\Phi_{k}(x-y)\right|+\left|\Phi_{k}(x)\right| d x \\
\leq & C B \sum_{a_{k+1}<|b y|^{-1}}\left|b a_{k+1} y\right| \int_{\mathbb{R}^{n}} \frac{\left(b a_{k+1}\right)^{n}}{\left(1+\left|b a_{k+1} x\right|\right)^{n+1}} d x \\
& +C B \sum_{a_{k+1} \geq|b y|^{-1}} \int_{|x|>2|y|} \frac{\left(b a_{k+1}\right)^{n}}{\left(\left|b a_{k+1} x\right|\right)^{n+1}} d x \\
\leq & C B \sum_{a_{k+1}<|b y|^{-1}}|b y| a_{k+1} \int_{\mathbb{R}^{n}} \frac{1}{(1+|x|)^{n+1}} d x \\
& +C B \sum_{a_{k+1} \geq|b y|^{-1}} \int_{2\left|b a_{k+1} y\right|}^{\infty} \frac{1}{r^{2}} d r \\
\leq & C B \sum_{a_{k+1}<|b y|^{-1}}|b y| a_{k+1}+C B \sum_{a_{k+1} \geq|b y|^{-1}} \frac{1}{|b y|} \frac{1}{a_{k+1}} .
\end{aligned}
$$

Let $k_{0}$ be the integer satisfying $a_{k_{0}}<|(a-1) y|^{-1} \leq a_{k_{0}+1}$. Then we have

$$
I \leq \frac{C B}{a_{k_{0}}} \sum_{k \leq k_{0}-1} a_{k+1}+C B a_{k_{0}+1} \sum_{k \geq k_{0}} \frac{1}{a_{k+1}} .
$$

From $a_{k+1} / a_{k} \geq a$ it follows that $a_{k+1} \leq a^{-1} a_{k+2} \leq \cdots \leq a^{k-k_{0}+1} a_{k_{0}}$ for $k \leq k_{0}$. Hence we get

$$
\sum_{k \leq k_{0}-1} a_{k+1} \leq \sum_{k \leq k_{0}-1} a_{k_{0}} a^{k-k_{0}+1}=a_{k_{0}} \sum_{k=0}^{\infty} \frac{1}{a^{k}}=a_{k_{0}} \frac{a}{a-1} .
$$

From $a_{k+1} / a_{k} \geq a$ it follows that $a_{k+1} \geq a a_{k} \geq \cdots \geq a^{k-k_{0}} a_{k_{0}+1}$ for $k \geq k_{0}$. Hence we get

$$
\sum_{k \geq k_{0}} \frac{1}{a_{k+1}} \leq \sum_{k \geq k_{0}} \frac{1}{a_{k_{0}+1}} \frac{1}{a^{k-k_{0}}}=\frac{1}{a_{k_{0}+1}} \sum_{k=0}^{\infty} \frac{1}{a^{k}}=\frac{1}{a_{k_{0}+1}} \frac{a}{a-1} .
$$

Thus we have

$$
I \leq C\left(\frac{a}{a-1}\right)^{n+2}
$$

If we define $\Phi_{j}^{1}$ by $\hat{\Phi}_{j}^{1}(\xi)=\eta\left(\xi / a_{j}\right)$, we get $\Phi_{j}^{1}=\Phi_{j-1}$. So, for $\Phi_{j}^{1}$ we have the same estimate as for $\Phi_{j}$. Therefore, we obtain

$$
\int_{|x|>2|y|}\left(\sum_{k \in \mathbb{Z}}\left|\Psi_{k}(x-y)-\Psi_{k}(x)\right|^{2}\right)^{1 / 2} d x \leq C\left(\frac{a}{a-1}\right)^{n+2} .
$$


Now let $\mathcal{B}_{1}=\mathbb{C}, \mathcal{B}_{2}=\ell^{2}$, define an $\ell^{2}$-valued function $\vec{K}(x)$ by $\vec{K}(x)=\left\{\Psi_{k}(x)\right\}_{k \in \mathbb{Z}}$, and the linear operator $\vec{T}$ by $\vec{T}(f)=\vec{K} * f$ for $f \in L^{\infty}\left(\mathbb{R}^{n}\right)$ with compact support. Then we have \|\|$\vec{T}(f)\left\|_{\mathcal{B}_{2}}\right\|_{L^{r}\left(\mathbb{R}^{n}\right)}=\left\|\left(\sum_{k \in \mathbb{Z}}\left|\Psi_{k} * f\right|^{2}\right)^{\frac{1}{2}}\right\|_{L^{r}\left(\mathbb{R}^{n}\right)}$, and so by Littlewood-Paley theory this is equivalent to $\|f\|_{L^{r}\left(\mathbb{R}^{n}\right)}$ for any $1<r<\infty$. By (A.7), the kernel $\vec{K}(x)$ satisfies the Hörmander condition. Thus, we can apply Proposition 4.6.4 in Grafakos [21], and get the conclusion of Lemma 2.6.

\section{Competing interests}

The author declares that he has no competing interests.

\section{Author's contributions}

The author contributed to the writing of this paper. He read and approved the final manuscript.

\section{Acknowledgements}

The work is partially supported by Grant-in-Aid for Scientific Research (C) (No. 23540228), Japan Society for the Promotion of Science.

Received: 12 December 2014 Accepted: 12 March 2015 Published online: 21 March 2015

\section{References}

1. Stein, EM: Problems in harmonic analysis related to curvature and oscillatory integrals. In: Proceedings of the International Congress of Mathematicians, Berkley, pp. 196-221 (1986)

2. Stein, EM, Wainger, S: Problems in harmonic analysis related to curvature. Bull. Am. Math. Soc. 84, 1239-1295 (1978)

3. Kim, W, Wainger, S, Wright, J, Ziesler, S: Singular integrals and maximal functions associated to surfaces of revolution. Bull. Lond. Math. Soc. 28, 291-296 (1996)

4. Fan, D, Pan, Y: A singular integral operator with rough kernel. Proc. Am. Math. Soc. 125, 3695-3703 (1997)

5. Fan, D, Pan, Y, Yang, D: A weighted norm inequality for rough singular integrals. Tohoku Math. J. 51, 141-161 (1999)

6. Xue, Q, Yabuta, K: On the boundedness of singular integrals with variable kernels. Tohoku Math. J. 61, 41-65 (2009)

7. Chen, J, Zhang, C: Boundedness of rough singular integrals on the Triebel-Lizorkin spaces. J. Math. Anal. Appl. 337, 1048-1052 (2008)

8. Chen, Y, Ding, Y: Rough singular integrals on Triebel-Lizorkin space and Besov space. J. Math. Anal. Appl. 347, 493-501 (2008)

9. Chen, Y, Ding, Y, Liu, H: Rough singular integrals supported on submanifolds. J. Math. Anal. Appl. 368, 677-691 (2010)

10. Grafakos, L, Stefanov, A: $L^{p}$ Bounds for singular integrals and maximal singular integrals with rough kernels. Indiana Univ. Math. J. 47, 455-469 (1998)

11. Fan, D, Sato, S: A note on singular integrals associated with a variable surface of revolution. Math. Inequal. Appl. 12(2), 441-454 (2009)

12. Li, W, Si, Z, Yabuta, K: Boundedness of singular integrals associated to surfaces of revolution on Triebel-Lizorkin spaces. Forum Math. (2014). doi:10.1515/forum-2014-0066

13. Bergh, J, Löfström, J: Interpolation Spaces: An Introduction. Springer, Berlin (1976)

14. Triebel, H: Theory of Function Spaces. Monogr. Math., vol. 78. Birkhäuser, Basel (1983)

15. Xue, Q, Yabuta, K: $L^{2}$ Boundedness Marcinkiewicz integrals along surfaces with variable kernels. Sci. Math. Jpn. 63, 369-382 (2006)

16. Jiang, Y, Lu, S: $L^{p}$ Boundedness of a class of maximal singular integral operators. Acta. Math. Sin. Chin. Ser. 35, 63-72 (1992) (in Chinese)

17. Lu, S, Taibleson, M, Weiss, G: Spaces Generated by Blocks. Publishing House of Beijing Normal University, Beijing (1989)

18. Lu, S: Applications of some block spaces to singular integrals. Front. Math. China 2(1), 61-72 (2007)

19. Colzani, L: Hardy spaces on sphere. PhD thesis, Washington University, St. Louis, MO (1982)

20. Ding, Y, Xue, Q, Yabuta, K: Boundedness of the Marcinkiewicz integrals with rough kernel associated to surfaces. Tohoku Math. J. 62, 233-262 (2010)

21. Grafakos, L: Classical and Modern Fourier Analysis. Pearson Education, Upper Saddle River (2004)

22. Sawano, Y, Yabuta, K: Fractional type Marcinkiewicz integral operators associated to surfaces. J. Inequal. Appl. 2014, $232(2014)$

23. Li, W, Yabuta, K: Rough singular integrals associated to submanifolds. Taiwan. J. Math. 16, 1557-1587 (2012) 